\title{
EL BOSQUE MESÓFILO DE MONTAÑA EN EL MUNICIPIO DE LANDA DE Matamoros, Querétaro, MÉxico
}

\author{
Sandra Cartujano ${ }^{1}$, Sergio Zamudio ${ }^{2}$, Othón Alcántara ${ }^{1}$ e Isolda luna ${ }^{1}$ \\ 'Departamento de Biología Evolutiva, Facultad de Ciencias, UNAM, Apartado Postal 70-399, México 04510, D.F., \\ México. Correo electrónico: ilv@hp.fciencias.unam.mx, \\ teléfono 56-22-48-32 \\ ${ }^{2}$ Instituto de Ecología A.C., Centro Regional del Bajío, Apartado Postal 386, 61600 Pátzcuaro, Michoacán, México
}

\begin{abstract}
Resumen: Se realizó un estudio florístico del bosque mesófilo de montaña localizado en el extremo oriental del municipio de Landa de Matamoros, Querétaro; el área está enclavada en la Sierra Madre Oriental y queda incluida en la Reserva de la Biosfera de la Sierra Gorda. En este municipio el bosque mesófilo se encuentra muy fragmentado, pero está más conservado principalmente en las localidades de Camarones, Neblinas, La Yesca y La Joya del Hielo. Se proporciona un listado florístico de las plantas vasculares, compuesto por 130 familias, 465 géneros y 774 especies. Se elaboró un mapa con la distribución de este tipo de vegetación en la región.

Palabras clave: bosque mesófilo de montaña, florística, Landa de Matamoros, Querétaro.

Abstract: A floristic inventory of the cloud forest of some localities of Landa de Matamoros municipality, Querétaro, was undertaken; this area is located in the Sierra Madre Oriental and is part of the Sierra Gorda Biosphere Reserve. In this zone the forest is fragmented, but it has a relatively good conservation status in some localities such as Camarones, Neblinas, La Yesca, and La Joya del Hielo. A floristic list of vascular plants composed by 130 families, 465 genera and 774 species was obtained. A distribution map of this vegetation type is included.
\end{abstract}

Keywords: cloud forest, floristics, Landa de Matamoros, Querétaro.

$\mathbf{E}$ 1 territorio de la República Mexicana posee una gran variedad de climas y una enorme diversidad fisiográfica; en él convergen, junto con la flora autóctona, elementos propios de las floras meridional y boreal, mismos que se han diversificado en múltiples ambientes y tipos de vegetación. Debido a ello, la flora mexicana es considerada como una de las más diversas del mundo. Actualmente se estima que la flora del país está compuesta por cerca de 30,000 especies de plantas vasculares (Toledo, 1988; Flores y Gerez, 1988).

Aunque no existe un registro suficientemente detallado y completo de la composición florística del país (Rzedowski, 1978), la experiencia de las últimas décadas ha sugerido que el número estimado de taxones reconocidos de la flora de México está aún por debajo del 90\%; de ahí la importancia de seguir haciendo estudios florísticos y de vegetación regionales que aporten información valiosa al inventario nacional.

El bosque mesófilo de montaña es un tipo de vegetación muy diverso que alberga gran cantidad de especies endémicas. El área ocupada por esta comunidad vegetal se está reduciendo rápidamente, como producto principalmente del crecimiento de la población humana y de la expansión de las actividades productivas como la agricultura, la ganadería, la extracción forestal y la influencia de otros factores como el fuego (Luna et al., 1988, 1989, 1994), que impiden la regeneración natural y traen como consecuencia el agotamiento de los recursos naturales y la pérdida de linajes evolutivos que no se encuentran en otros sitios (Halffter, 1992).

En el estado de Querétaro el bosque mesófilo de montaña cubre una superficie aproximada de $54 \mathrm{~km}^{2}$, lo que representa menos del $0.5 \%$ de su territorio. Esta comunidad vegetal se establece en cañadas o laderas húmedas, distribuidas entre 800 y 2750 m de altitud; la mayor parte de estas áreas están ubicadas en la vertiente de barlovento de la Sierra Madre Oriental, donde la influencia de los vientos alisios del norte es más notoria. Se distribuye parcialmente en los municipios de Jalpan, Landa de Matamoros, Pinal de Amoles y San Joaquín. Sin embargo, los bosques más extensos y continuos de esta comunidad vegetal se encuentran en el extremo orien- 


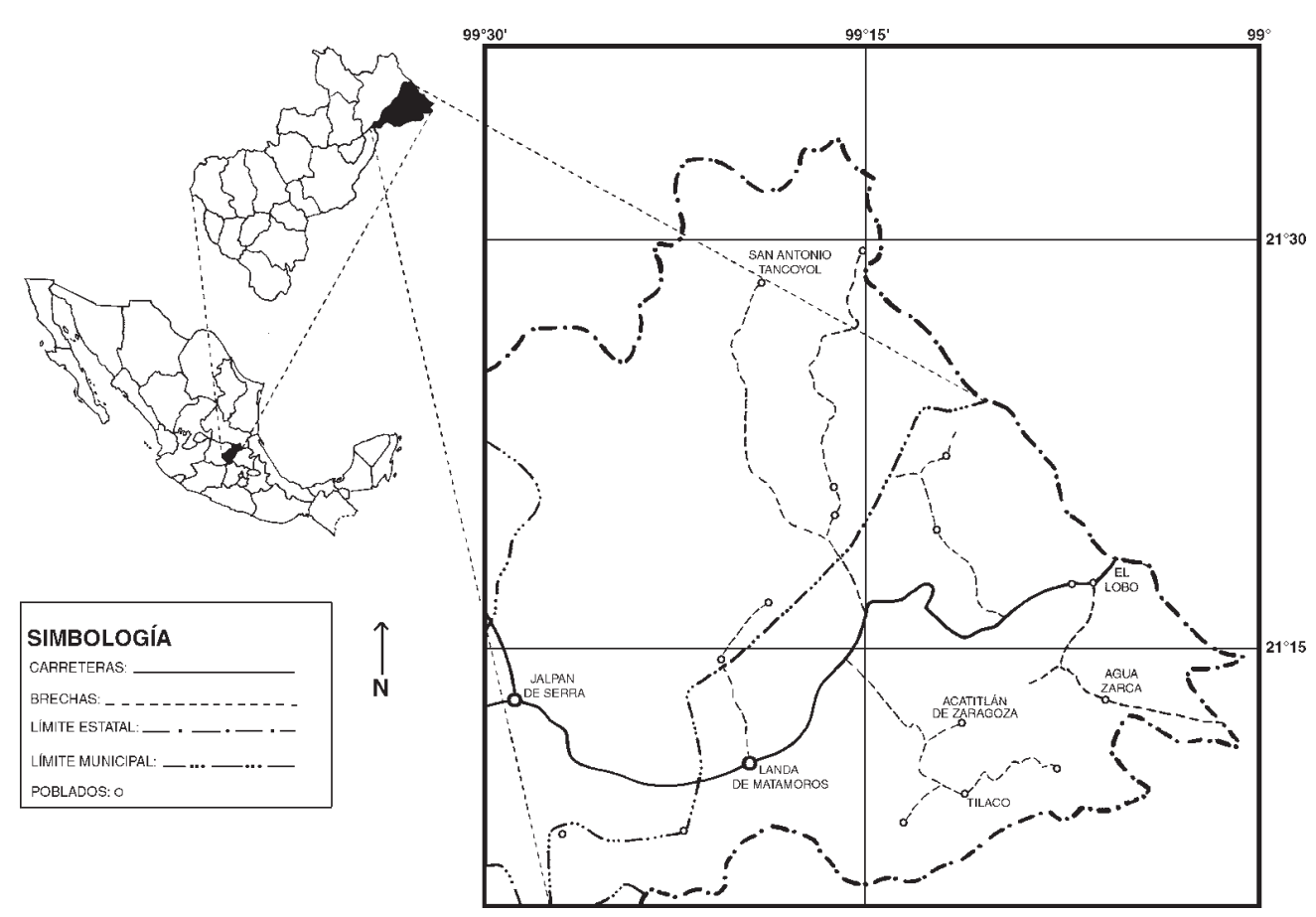

Figura 1. Localización del área de estudio.

tal del municipio de Landa de Matamoros, por lo que se seleccionó esta región como área de estudio (Zamudio y Fernández, 1990; Zamudio et al., 1992; INEGI, 1992).

Entre los estudios florísticos y de vegetación más importantes que proporcionan información sobre el bosque mesófilo de montaña en Querétaro se encuentran los de Puig (1976) sobre la vegetación de la Huasteca, en donde se incluyen los municipios de Landa de Matamoros, Jalpan, Cadereyta y San Joaquín; el de Zamudio (1984) sobre la vegetación de la cuenca del río Estórax; el de Argüelles et al. (1991), quienes proporcionan un listado preliminar de especies para el estado; el de Zamudio y Fernández (1990), quienes llevan a cabo un estudio preliminar del bosque mesófilo de montaña en Querétaro, en el que registran 635 especies; el de Zamudio et al. (1992) en el que se describe la vegetación del estado; el de Díaz-Barriga y Palacios-Ríos (1992) sobre los helechos de Querétaro; y el de Arreguín et al. (1997) sobre la flora estatal, entre los más importantes. Por otra parte, desde 1985 a la fecha se han realizado intensas campañas de exploración y colecta de ejemplares botánicos en todo el estado, en el marco del proyecto denominado "Flora del Bajío y de Regiones Adyacentes", que han ayudado a incrementar el conocimiento de la flora del estado.

La finalidad del presente trabajo es contribuir al conocimiento de los bosques montanos de México, persiguiendo los siguientes objetivos: conocer la composición florística del bosque mesófilo de montaña del municipio de Landa de Matamoros, Querétaro; hacer una caracterización del bosque mesófilo de varias localidades dentro del municipio; llevar a cabo un análisis geográfico de las familias y géneros de plantas vasculares y realizar un mapa que muestre las zonas en las que se distribuye este tipo de vegetación en Landa de Matamoros; y por último, discutir algunos aspectos sobre su conservación y uso sustentable.

\section{Área de estudio}

El municipio de Landa de Matamoros se encuentra en el extremo noreste del estado de Querétaro, entre los $21^{\circ} 06^{\prime}$ y $21^{\circ} 27^{\prime}$ de latitud norte y $\operatorname{los} 99^{\circ} 02^{\prime}$ y $99^{\circ} 27^{\prime}$ de longitud oeste. Limita al noreste y este con el estado de San Luis Potosí, al sur con el estado de Hidalgo y al norte y oeste con el municipio de Jalpan. Las localidades de estudio se encuentran entre 800 y $2200 \mathrm{~m}$ de altitud, en los cañones del río Tancuilín y de otros arroyos que desembocan en el río Moctezuma, en las cercanías de Agua Zarca, El Humo, Neblinas y Río Verdito (Zamudio y Fernández, 1990; figura 1. El área de estudio queda incluida en la región denominada localmente como Sierra Gorda, la que fue decretada como Reserva de la Biosfera el 14 de mayo de 1997 por el Poder Ejecutivo Federal y la Secretaría de Medio Ambiente, Recursos Naturales y Pesca (Diario Oficial de la Federación, 1997).

El municipio de Landa se encuentra en la provincia fisiográfica de la Sierra Madre Oriental, en la subprovincia del Carso Huasteco (INEGI, 1992). Es una región eminentemente montañosa, formada por un conjunto de serranías alargadas, con orientación noroeste-sureste (Consejo de Recursos Minerales, 1992). La topografía del área es muy accidentada, con predominancia de cerros. 
La zona de estudio pertenece a la Región Hidrológica del río Pánuco y en particular a la cuenca del río Moctezuma, que en esta parte de su recorrido marca el límite entre los estados de Hidalgo y Querétaro. La porción norte de la zona está irrigada principalmente por el río Tancuilín y la parte sur por los ríos San Juan y Tangojó.

En el área de estudio afloran rocas sedimentarias muy antiguas, las cuales pertenecen al Jurásico Superior y al Cretácico. Los suelos del área de estudio se derivan de rocas sedimentarias, fundamentalmente calizas, aunque también intervienen lutitas, areniscas y conglomerados. La mayoría de los suelos que se han originado aquí son residuales y no tienen gran desarrollo, y por lo tanto son poco profundos. Los litosoles están ampliamente representados en el municipio y se encuentran en las partes más altas de la sierra y en las laderas de mayor pendiente; están asociados principalmente con rendzinas y regosoles que son más profundos y se desarrollan en lugares con menor pendiente (INEGI, 1992). La mayoría de los suelos no son recomendables para uso agrícola, ya que fácilmente se erosionan.

El bosque mesófilo de montaña se desarrolla en la zona más húmeda del estado; debido a su ubicación en la vertiente oriental de la Sierra Madre, la región está expuesta a la influencia directa de los vientos húmedos del noreste provenientes del Golfo de México, los que al chocar con los macizos montañosos producen abundantes precipitaciones, así como la presencia de neblina y humedad ambiental muy alta durante la mayor parte del año (Zamudio et al., 1992).

En la carta de climas del Atlas Nacional del Medio Físico (Anónimo, 1981) la mayor parte del área queda incluida dentro del clima semicálido húmedo, con lluvias todo el año, con precipitación del mes más seco mayor de $40 \mathrm{~mm}$ y porcentaje de lluvia invernal menor de $18 \%$, que corresponde a la fórmula $(\mathrm{A}) \mathrm{C}(\mathrm{fm})$, de acuerdo con el sistema de clasificación climática de Köppen modificado por García (1988) y que se comparte con la región cercana de Chapulhuacán, Hidalgo. Es muy probable también que en la zona se presente un clima templado húmedo con lluvias todo el año, con precipitación del mes más seco mayor de $40 \mathrm{~mm}$ y porcentaje de lluvia invernal menor de $18 \%$ $(\mathrm{C}(\mathrm{fm})$ ), propio de la vecina población de Xilitla, San Luis Potosí. Por su parte, CONABIO-Estadigrafía (1977) cita un clima semicálido subhúmedo, con lluvias en verano, el más húmedo de los subhúmedos, que corresponde a la fórmula $\mathrm{A}(\mathrm{C}) \mathrm{w}_{2}(\mathrm{w})$; lo anterior hace evidente que en el área se presenta un gradiente climático en el que influyen de manera directa cambios bruscos de altitud y de exposición.

\section{Métodos}

Los sitios de colecta se eligieron procurando que la vegetación estuviera lo mejor conservada posible, de acuerdo a visitas de prospección a la zona, y con base en los siguientes mapas: carreteras (Secretaría de Comunicaciones y Transportes, 1986), topografía a escala 1:250,000 (INEGI, 1981; INEGI, 1995a), uso del suelo y vegetación a escala 1:250,000 (INEGI, 1985), así como en material aerofotográfico escala 1:75,000 (INEGI, 1995b).

Se realizaron 14 salidas de campo iniciando en marzo de 1998 hasta abril de 2000, con una duración de cuatro a cinco días cada una. En cada salida se recolectaron especímenes botánicos en estado de floración y/o fructificación, y se anotaron los datos que permitieran describir la zona de estudio. La información obtenida se capturó en una base de datos en formato Access 2000. Asimismo, se consultó la colección de plantas y la base de datos del herbario del Centro Regional del Bajío del Instituto de Ecología, A.C. (IEB), de la cual se obtuvo una lista de las especies del bosque mesófilo del estado de Querétaro. Aunque los objetivos de este trabajo no incluyen llevar a cabo un análisis de la vegetación, se elaboraron algunos perfiles vegetacionales mediante la consulta de las bases de datos; con ello fue posible determinar las especies arbóreas más abundantes en los diferentes pisos altitudinales. Asimismo, se llevaron a cabo algunos levantamientos de la vegetación siguiendo la escuela de Zürich-Montpellier modificada por Mueller-Dombois et al. (1988), para conocer de manera general su estructura y diversidad.

El material colectado fue herborizado de acuerdo con los procedimientos botánicos convencionales (Lot y Chiang, 1986) y posteriormente identificado a nivel de especie, con la ayuda de especialistas para algunas familias. El ordenamiento de las familias se hizo de acuerdo con el sistema de Engler y Diels (1936) en el caso de angiospermas y gimnospermas, y con la clasificación propuesta por Mickel y Beitel (1988) para pteridofitas y grupos afines. Los autores de las especies se abreviaron de acuerdo con Brummitt y Powell (1992). La distribución actual de las familias y de los géneros se obtuvo a partir del trabajo de Mabberley (1997). Los especímenes se cotejaron en las colecciones del Herbario de la Facultad de Ciencias (FCME), el Herbario Nacional del Instituto de Biología (MEXU) y el Herbario del Centro Regional del Bajío del Instituto de Ecología, A.C. (IEB). Los ejemplares están depositados en el Herbario de la Facultad de Ciencias, UNAM (FCME).

Se elaboró un mapa para mostrar la distribución de las áreas conservadas del bosque mesófilo de montaña en el municipio de Landa de Matamoros, mediante la interpretación por medio de estereoscopios del material aerofotográfico, escala 1:75,000 (INEGI, 1995b), marcando las unidades de vegetación correspondientes al bosque mesófilo de montaña, para posteriormente restituirlos con la ayuda del stereosketch sobre la carta topográfica escala 1:50,000 (INEGI, 1981). Se digitalizaron las curvas maestras cada $100 \mathrm{~m}$ del mapa topográfico del área a escala 1:500,000 con el Sistema de Información Geográfica ILWIS, versión 2.1 (International 


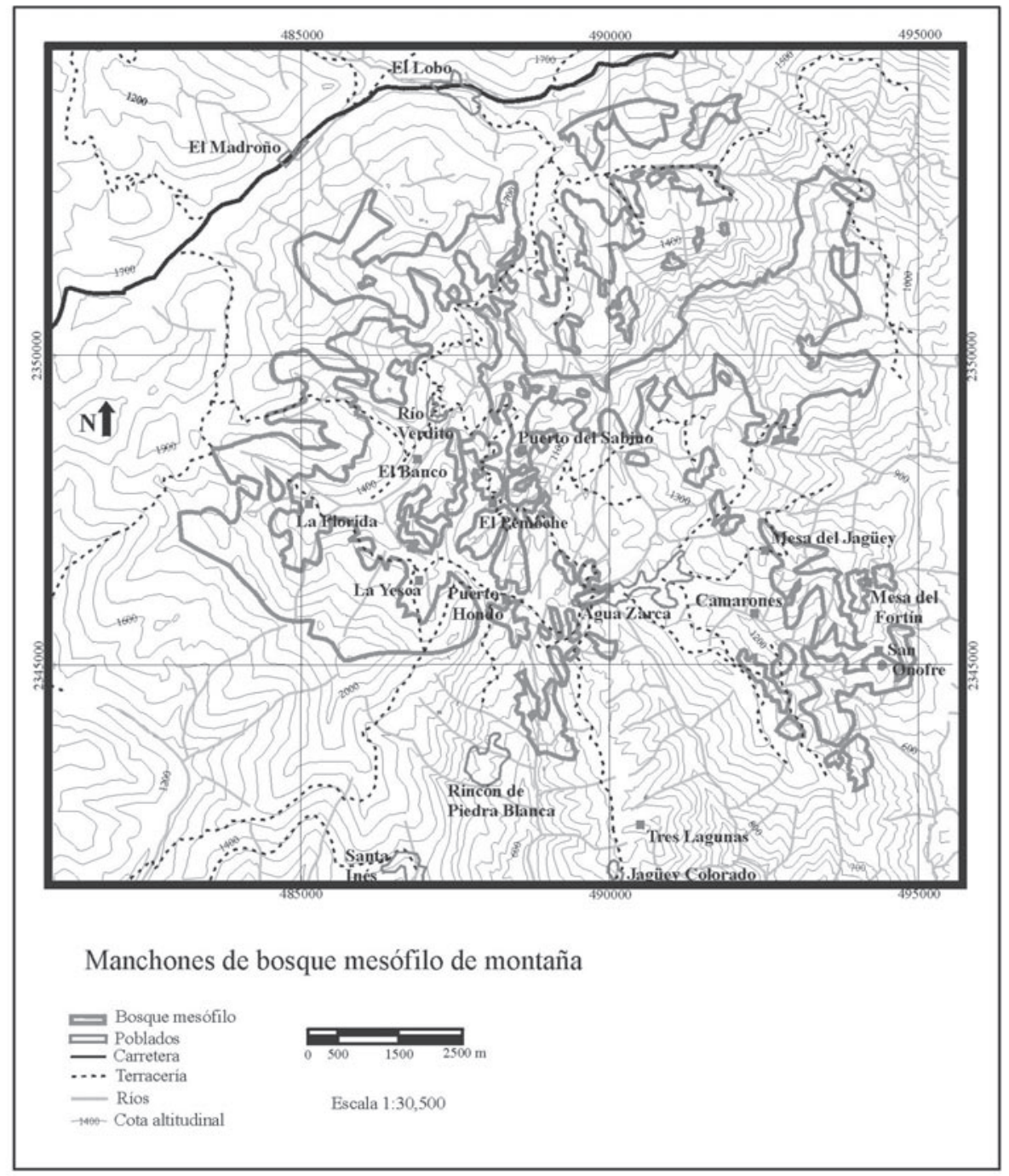

Figura 2. Mapa topográfico donde se muestran los principales manchones de bosque mesófilo de montaña de Landa de Matamoros, Querétaro. Las unidades UTM en el mapa corresponden a la zona 14-N.

Institute for Aerospace Survey and Earth Sciences, 1997) y las unidades de bosque mesófilo de montaña para elaborar el mapa de distribución.

Los datos geográficos obtenidos en cada salida a campo se capturaron como un archivo de puntos que se desplegaron sobre el mapa para identificar las unidades correspondientes a bosque mesófilo.

\section{Resultados}

Caracterización del bosque. El bosque mesófilo de montaña del municipio de Landa de Matamoros se encuentra muy fragmentado y perturbado; las áreas conservadas de mayor extensión persisten en cañadas protegidas y en laderas escarpadas y de difícil acceso a lo largo del cañón del río Tancuilín, principalmente en las inmediaciones de Neblinas y El Humo. Otras áreas poco alteradas se encuentran en la parte alta de los cerros, incluyendo La Joya del Hielo y una franja irregular que se extiende por arriba de La Florida y La Yesca hasta Puerto Hondo. En las otras localidades estudiadas el bosque está muy alterado y pequeños grupos de árboles alternan con parcelas de cultivo y extensos potreros (figura 2).

En los sitios mejor conservados el bosque mesófilo de montaña es muy diverso y exuberante y está formado por numerosas especies de árboles distribuidos en varios estratos. La densidad de los árboles y la sobreposición de su follaje forman un dosel denso que produce un ambiente con penumbra y alta humedad en su interior. Las plantas epífitas que cubren los troncos y las ramas de los árboles son abundantes, así como también las trepadoras leñosas.

Los árboles más importantes del dosel superior son corpulentos, pueden llegar a medir 30 ó $40 \mathrm{~m}$ de altura y pierden total o parcialmente las hojas durante la estación seca y fría del año. Entre éstos, los más notorios son: Aphananthe 
monoica, Liquidambar macrophylla, Persea americana, Quercus affinis, $Q$. germana, $Q$. rysophylla, $Q$. sartorii, $Q$. xalapensis, Robinsonella discolor y Ulmus mexicana.

Siguiendo un gradiente climático y altitudinal, se pueden observar los siguientes cambios en la composición del bosque (figura 3):
En la parte baja, entre 600 y 800 m s.n.m., los elementos del bosque mesófilo se mezclan con los del bosque tropical subperennifolio, con el que forma una franja de transición. Esta condición se aprecia en el fondo del cañón del río Tancuilín y en los alrededores de San Onofre y Rancho Nuevo, en el camino entre Agua Zarca y Pisaflores, Hidalgo,

a)

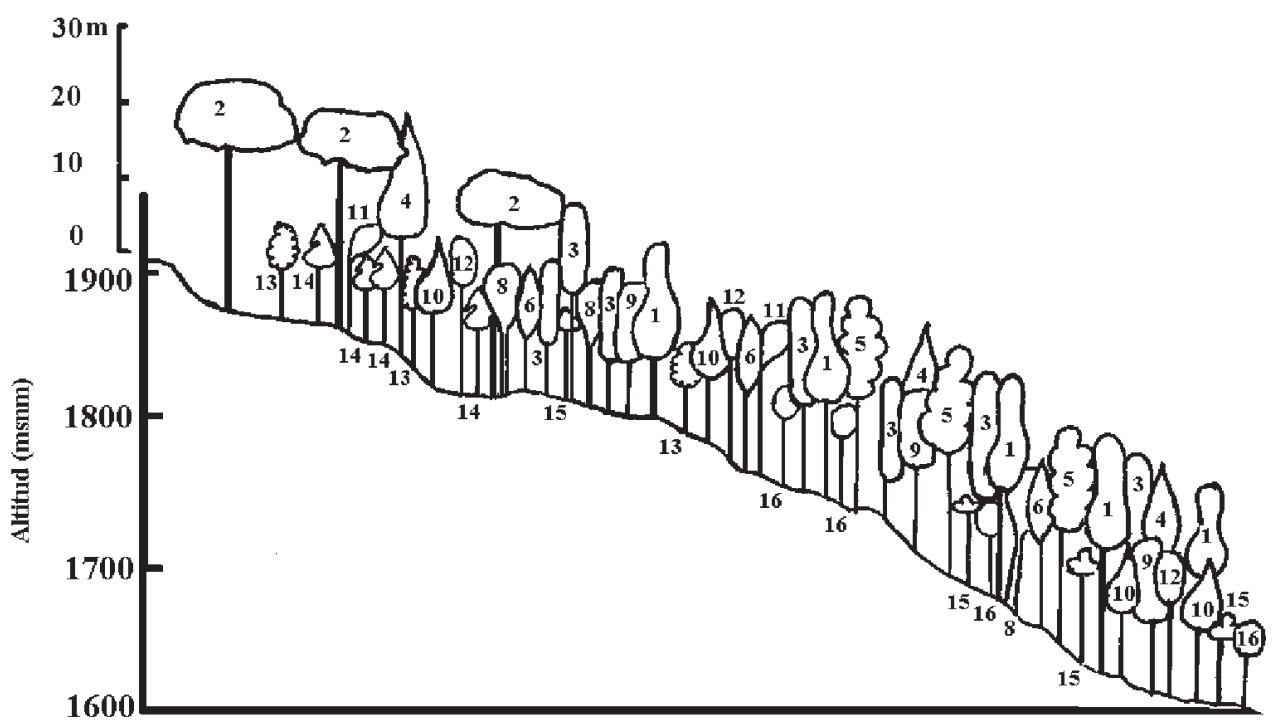

b)

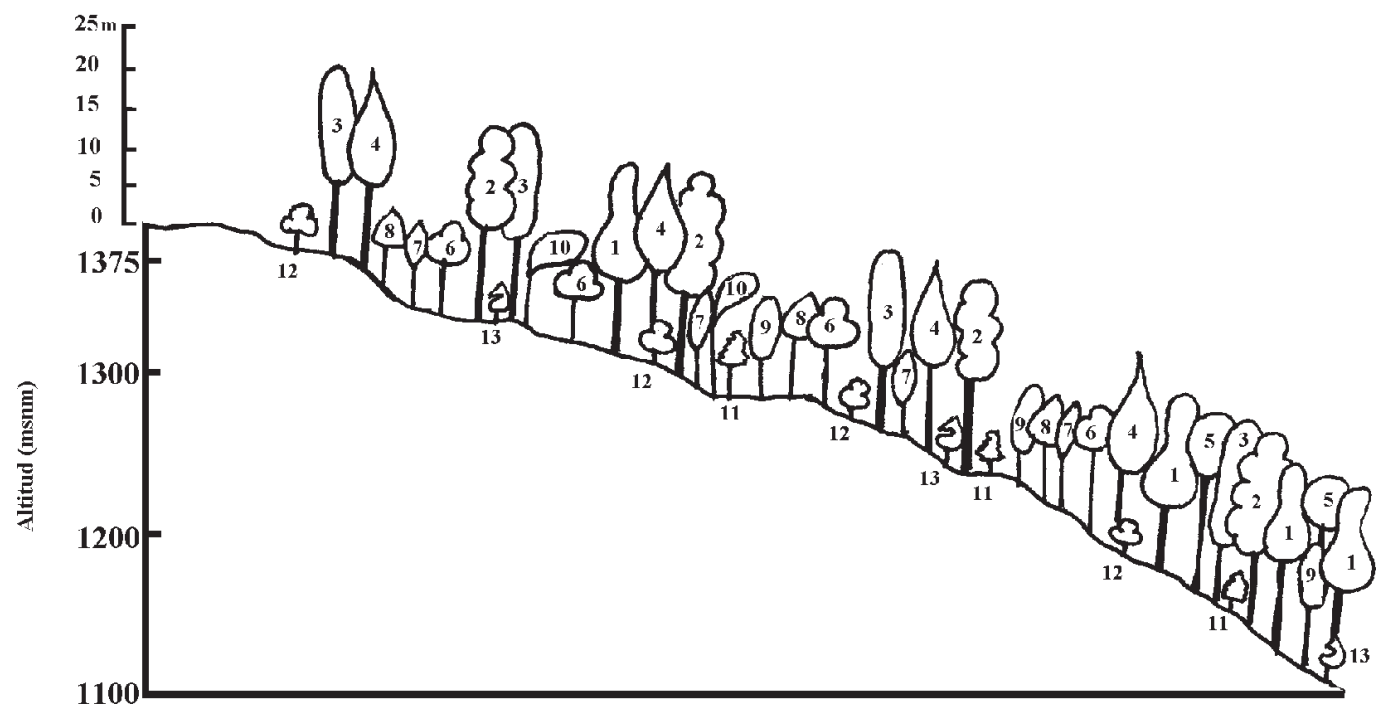

Figura 3. Perfiles esquemáticos de dos localidades de bosque mesófilo de montaña con sus especies representativas en cada localidad. a) La Yesca (orientación noroeste): 1. Liquidambar macrophylla, 2. Cupressus lusitanica, 3. Quercus affinis, 4. Quercus polymorpha, 5. Quercus sartorii, 6. Cercis canadensis, 7. Quercus sp., 8. Quercus eugeniifolia, 9. Quercus aff. pinnativenulosa, 10. Ostrya virginiana , 11. Tilia mexicana, 12. Cornus excelsa, 13. Eugenia xalapensis, 14. Magnolia dealbata, 15. Ternstroemia sylvatica y 16. Vaccinium leucanthum. b) Camarones (orientación oeste) 1. Liquidambar macrophylla, 2. Persea americana, 3. Quercus rysophylla, 4. Quercus germana, 5. Quercus sartorii, 6. Clethra kenoyeri, 7. Clethra pringlei, 8. Dalbergia palo-escrito, 9. Quercus polymorpha, 10. Daphnopsis mollis, 11. Rapanea myricoides, 12. Reevesia clarki y 13. Ternstroemia huasteca. 
donde el estrato superior está formado por árboles de 30 a 40 $\mathrm{m}$ de altura, entre los que destacan: Aphananthe monoica, Ficus pertusa, Persea americana, Quercus rysophylla, Ulmus mexicana y Zuelania guidonia.

El estrato arbóreo medio lo constituyen árboles de 15 a 25 $\mathrm{m}$ de altura, como Cupania dentata, Reevesia clarkii y Sapindus saponaria.

El estrato arbóreo bajo, de 6 a $12 \mathrm{~m}$ de alto, es muy diverso y está formado por numerosas especies, entre las que se han registrado Bernardia interrupta, Cinnamomum pachypodum, Clethra pringlei, Dendropanax arboreus, Eugenia capuli, Gymnanthes longipes, Heliocarpus americanus, $H$. appendiculatus, $H$. mexicanus, Inga lactibracteata, Nectandra salicifolia, $N$. sanguinea, Persea liebmannii, Pithecellobium insigne, Ternstroemia huasteca, Trema micrantha, Trichilia havanensis, Trophis racemosa y Wimmeria concolor.

El estrato arbustivo también es diverso y más o menos denso y está compuesto por arbustos de 2 a $6 \mathrm{~m}$ de altura, entre los que se encuentran Acalypha schlechtendaliana, Annona globiflora, Ardisia escallonioides, Bocconia frutescens, Bumelia verruculosa, Callicarpa acuminata, $C$. pringlei, Chiococca alba, C. pachyphylla, Chione mexicana, Chrysophyllum mexicanum, Conostegia xalapensis, Cordia podocephala, Exothea copalillo, Gouania polygama, Hamelia patens, Myrica cerifera, Parathesis brevipes, Picramnia antidesma, Piper auritum, Pothomorphe umbellata, Prunus samydoides, Psychotria erythrocarpa, $P$. microdon, Tabernaemontana alba, Ternstroemia huasteca, Urera alceifolia y Zapoteca portoricensis.

En este bosque las plantas trepadoras, tanto herbáceas como leñosas, son abundantes y se encuentran representadas por las siguientes especies: Celtis iguanaea, Cissampelos pareira, Gonolobus niger, Marsdenia pringlei, Mikania cordifolia, Pachyrhizus erosus, Parthenocissus quinquefolia, Smilax aristolochifolia, S. jalapensis, S. mollis, Teramnus uncinatus, Trichostigma octandrum y Xylosma flexuosum.

Las plantas epífitas crecen formando grupos densos sobre los troncos y las ramas de los árboles; entre ellas se encuentran varias especies de helechos y de orquídeas, como: Campyloneurum phyllitidis, Lycaste consobrina, Maxillaria densa, Nidema boothii, Pecluma plumula, Peperomia microphylla, P. quadrifolia, Phlebodium areolatum, Pleurothallis nicaraguensis, Polypodium furfuraceum y Rhipsalis baccifera.

Las plantas herbáceas que crecen en este bosque también son abundantes. Entre ellas se encuentran numerosos helechos, compuestas, begonias y representantes de otras familias, como: Achimenes grandiflora, Adiantum concinnum, A. tenerum, A. trapeziforme, Anemia mexicana var. mexicana, Asplenium cuspidatum, Begonia franconis, B. glandulosa, Blechnum brownei, B. glandulosum, Encyclia cochleata, Gronovia scandens, Heliconia schiedeana, Laelia anceps, Lygodium venustum, Malaxis histionantha,
Maxillaria variabilis, Oplismenus compositus, Petrea volubilis, Phanerophlebia remotispora, Psychotria pubescens, Russelia maculosa, R. syringaefolia, Sedum retusum, Selaginella extensa, S. stenophylla, Spiranthes costaricensis, Tectaria heracleifolia, Tibouchina naudiniana, Tripogandra serrulata y Vernonia arctioides.

Entre 800 y $1400 \mathrm{~m}$ de altitud el bosque mesófilo adquiere su máxima expresión en diversidad y estructura; se desarrolla en laderas muy inclinadas del cañón del río Tancuilín, en las inmediaciones de Neblinas, El Humo, Agua Zarca, Camarones, El Naranjo y El Pemoche. En el estrato arbóreo alto destaca la presencia de árboles corpulentos que superan los $30 \mathrm{~m}$ de altura; los más notorios son Ulmus mexicana, Quercus germana y $Q$. rysophylla, además de Liquidambar macrophylla, Persea americana, Quercus affinis, Q. sartorii, Q. xalapensis y Reevesia clarkii.

En el estrato arbóreo medio, que va de 15 a $25 \mathrm{~m}$ de alto, se encuentran: Aphananthe monoica, Cinnamomum effusum, Inga huastecana, Photinia mexicana, Quercus polymorpha, Reevesia clarkii, Robinsonella discolor, Sapindus saponaria, Senna racemosa y Wimmeria concolor.

En el estrato arbóreo bajo, de 6 a $12 \mathrm{~m}$ de alto, se registra la presencia de Bauhinia chapulhuacania, Beilschmiedia mexicana, Bernardia interrupta, Boehmeria ulmifolia, Cinnamomum bractefoliaceum, C. pachypodum, Clethra kenoyeri, C. pringlei, Cleyera theaeoides, Daphnopsis mollis, Eugenia crenularis, E. xilitlensis, Forestiera reticulata, Gymnanthes longipes, Heliocarpus americanus, Inga huastecana, I. lactibracteata, Licaria campechiana, Myrcianthes fragrans, Nectandra salicifolia, $N$. sanguinea, Ocotea klotzschiana, O. psychotrioides, Perrottetia ovata, Persea liebmanii, Photinia mexicana, Picramnia xalapensis, Pithecellobium insigne, Rapanea myricoides, Reevesia clarkii, Saurauia scabrida, Senna racemosa var. moctezumae, Tridimeris hahniana, Turpinia occidentalis, Viburnum sp. y Zanthoxylum hidalgense.

El estrato arbustivo, de 2 a $6 \mathrm{~m}$ de altura, es denso y está formado por: Acalypha schlechtendaliana, Ardisia escallonioides, Bocconia frutescens, Chiococca pachyphylla, Chione mexicana, Clerodendrum bungei, Conostegia xalapensis, Daphnopsis mollis, Dennstaedtia globulifera, Elaeodendron trichotomus, Euonymus acuminatus, Hamelia patens, Hydrangea aff. nebulicola, Ilex condensata, Kearnemalvastrum lacteum, Leandra cornoides, Malvaviscus arboreus, Miconia anisotricha, M. moorei, Myriocarpa longipes, Oyedaea ovalifolia, Picramnia xalapensis, Piper auritum, Pothomorphe umbellata, Psychotria erythrocarpa, P. graciliflora, P. tenuifolia, Tournefortia hirsutissima, Trichilia hirta, Urera alceifolia, U. caracasana y Zanthoxylum elegantissimum.

Entre las plantas trepadoras se han registrado las siguientes: Bomarea hirtella, Cissampelos pareira, Cissus sicyoides, Clematis pitcheri, Gelsemium sempervirens, Matelea velutina, Parthenocissus quinquefolia, Passiflora serratifolia, 
Smilax aristolochiifolia, S. domingensis, S. glauca, S. mollis, S. jalapensis, Solanum appendiculatum, S. refractum, S. seaforthianum, Toxicodendron radicans, Valeriana scandens y Xylosma flexuosum.

En este bosque las plantas epífitas son abundantes y éstas están representadas principalmente por varias especies de helechos, orquídeas, piperáceas y crasuláceas, entre las que se pueden mencionar a: Catasetum integerrimum, Isochillus unilateralis, Nidema boothii, Pecluma plumula, Peperomia microphylla, P. quadrifolia, Phlebodium areolatum, Pleopeltis crassinervata, $P$. interjecta, $P$. fallax, Polypodium furfuraceum, $P$. polypodioides var. aciculare, $P$. rhodopleuron, Stanhopea tigrina, Tillandsia bartramii y Trichocentrum candidum.

Las plantas herbáceas son muy abundantes. Entre ellas destacan gran variedad de helechos de los géneros Adiantum, Anemia, Asplenium, Campyloneurum, Cheilanthes, Ctenitis, Cystopteris, Dennstaedtia, Diplazium, Pteris y Thelypteris. En este estrato sobresalen también por su importancia las orquídeas y otras plantas como: Achimenes grandiflora, Ascyrum hypericoides, Asplenium cuspidatum, Begonia franconis, B. glabra, B. xilitlensis, Callaeum septentrionale, Campyloneurum angustifolium, Chamaedorea microspadix, Coccocypselum guianense, Dichaea neglecta, Echeveria rosea, Elaphoglossum glaucum, Encyclia cochleata, E. mariae, Epidendrum propinquum, Fleischmannia pycnocephala, Fernaldia pandurata, Heliconia schiedeana, Holodictyum ghiesbregthii, Lactuca graminifolia var. mexicana, Maxillaria variabilis, Melasma physalodes, Mimophytum omphalodoides, Ocimum micranthum, Arthrostemma ciliatum, Peperomia blanda, Phanerophlebia nobilis, Pseudelephantopus spicatus, Sclerocarpus uniserialis var. frutescens, Selaginella delicatissima, S. extensa, S. harrisii, S. stenophylla, Smithiantha zebrina y Tectaria heracleifolia. En este estrato destaca la presencia de Helosis cayennensis var. mexicana, planta parásita o hemiparásita de color rojo brillante. Es posible que la presencia de varias de las plantas herbáceas registradas se vea favorecida por la perturbación y la alta humedad ambiental, lo que permite la existencia de una mayor diversidad en este estrato que en los bosques no perturbados.

Entre 1400 y $1800 \mathrm{~m}$ de altitud, en los alrededores de El Aguacate, El Calvario, La Florida, Río Verdito y La Yesca, el bosque mesófilo cambia en su composición florística y es menos denso y menos alto. En este bosque se distinguen tres estratos arbóreos; un estrato arbóreo alto de 25 a $30 \mathrm{~m}$, compuesto principalmente por Liquidambar macrophylla, Clethra kenoyeri, Persea americana, Quercus affinis, $Q$. polymorpha y $Q$. sartorii. En el estrato arbóreo medio, de 15 a 20 m, se registran: Carya ovata var. mexicana, Cercis canadensis, Cinnamomum effusum, Ostrya virginiana, Prunus serotina subsp. serotina, Styrax glabrescens, Tilia mexicana y Turpinia occidentalis, y en el estrato arbóreo bajo, de 8 a 15 m, predominan: Cinnamomum salicifolium, Clethra pringlei, Cleyera theaeoides, Cornus disciflora, C. excelsa, Dalbergia palo-escrito, Daphnopsis mollis, Eugenia xalapensis, Garrya laurifolia, Gymnanthes longipes, Ilex decidua, I. discolor, Juglans mollis, Litsea glaucescens, Lonchocarpus rugosus, Ocotea klotzchiana, Perrottetia ovata, Saurauia scabrida, Ternstroemia sylvatica y Wimmeria concolor.

El estrato arbustivo, de 2 a $3 \mathrm{~m}$ de alto, está compuesto por Archibaccharis schiedeana, Brickellia glandulosa, Cestrum oblongifolium, Chiococca alba, Choisya ternata, Clerodendrum bungei, Eupatorium rivale, Forestiera reticulata, Heberdenia penduliflora, Malvaviscus arboreus, Parathesis leptopa, Psychotria graciliflora, Solanum hispidum y Tibouchina naudiniana.

Los bejucos y plantas trepadoras herbáceas más conspicuas son: Bomarea hirtella, Canavalia villosa, Cissampelos pareira, Clematis pitcheri, Lonicera pilosa, Matelea velutina, Passiflora sicyoides, Parthenocissus quinquefolia, Smilax aristolochiifolia, S. mollis, Vitis bourgaeana y Xylosma flexuosum.

Entre las plantas epífitas se encuentran: Aporocactus martianus, Asplenium praemorsum, Campyloneurum angustifolium, Epidendrum longipetalum, E. propinquum, Isochilus unilateralis, Pecluma cyaticola, Pleopeltis mexicana, Polypodium fraternum, $P$. furfuraceum, $P$. plebeium, P. plesiosorum, P. polypodioides var. aciculare, Stanhopea tigrina y Tillandsia bartramii.

Algunas de las plantas herbáceas que se han encontrado en este bosque son: Aldama dentata, Arundinella deppeana, Begonia franconis, B. gracilis, Bidens alba var. radiata, Cheilanthes aemula, Coulterophytum brevipes, Cyclopogon luteo-albus, Cystopteris fragilis, Dennstaedtia cicutaria, Deppea purpusii, Diplazium franconis, Dryopteris wallichiana, Duchesnea indica, Echeveria rosea, Galium orizabae, Lepechinia schiedeana, Lobelia berlandieri, L. divaricata, L. laxiflora, L. tarsophora, Maurandya erubescens, Mildella intramarginallis, Physalis gracilis, $P$. pubescens, Pinguicula moranensis, Ponthieva ephippium, Pteris cretica, Salvia puberula, Sanicula liberta, Scleria oligantha, Sedum retusum, Selaginella extensa, S. harrisii, S. stenophylla, Smithiantha zebrina y Spananthe paniculata.

En este intervalo altitudinal el bosque se encuentra fuertemente perturbado y en grandes extensiones ha sido desplazado por los potreros y áreas de cultivo, por ejemplo: en el camino entre El Lobo y Río Verdito y en las inmediaciones de Puerto del Sabino, El Sabinito y San Juan, el bosque ha sido devastado y sólo permanece en laderas de difícil acceso, en donde se aprecian varios rodales de Liquidambar macrophylla. En La Florida y Puerto de Guadalupe las laderas deforestadas se destinan a potreros y sólo en pequeñas áreas inaccesibles se encuentran algunas especies arbóreas.

Las comunidades densas, casi uniespecíficas de Liquidambar macrophylla presentes en este intervalo altitudinal representan diferentes etapas de la regeneración del bosque, ya que el liquidámbar es un árbol de rápido crecimiento que 
resiste a las condiciones de disturbio, por lo que prevalece en la primeras etapas de la sucesión del bosque mesófilo de montaña en el área.

Por arriba de los $1800 \mathrm{~m}$ de altitud y hasta los $2100 \mathrm{~m}$ en La Yesca y La Joya del Hielo, el bosque mesófilo se caracteriza por la presencia de Cupressus lusitanica como árbol codominante en el estrato arbóreo alto, de 20 a $30 \mathrm{~m}$, junto con Carpinus caroliniana, Ostrya virginiana, Pinus greggii, Prunus serotina spp. serotina, Quercus affinis, $Q$. sartorii y Tilia mexicana. Formando un estrato arbóreo medio 15 a $20 \mathrm{~m}$ se encuentran varias especies como: Cercis canadensis, Magnolia dealbata, M. schiedeana y Persea americana, y el estrato arbóreo bajo, de 6 a 15 m, lo forman Cercocarpus macrophyllus, Cinnamomum salicifolium, Clethra kenoyeri, Cleyera theaeoides, Cornus disciflora, $C$. excelsa, Garrya laurifolia, Ilex discolor, Ocotea klotzchiana, Perrottetia ovata, Picramnia xalapensis, Rapanea myricoides, Rhamnus serrata, Styrax glabrescens, Taxus globosa, Ternstroemia sylvatica y Vaccinium leucanthum.

El estrato arbustivo está formado por Archibaccharis schiedeana, Baccharis lancifolia, Ceanothus coeruleus, Choisya ternata, Decatropis bicolor, Ageratina ligustrina, Forestiera reticulata, Heberdenia penduliflora, Lamourouxia multifida, Litsea glaucescens, Malvaviscus arboreus, Oxyrhynchus volubilis, Rhus aromatica var. trilobata, Roldana angulifolia, Senecio lanicaulis, Stevia pilosa, Xylosma flexuosum y Zanthoxylum elegantissimum.

En este intervalo altitudinal las plantas trepadoras disminuyen en densidad y diversidad. Entre ellas destacan: Bomarea hirtella, Clematis pitcheri, Lonicera pilosa y Smilax mollis; las epífitas más conspicuas son: Aporocactus martianus, Asplenium praemorsum, Hymenophyllum tunbrigense, Pecluma cyathicola, Peperomia quadrifolia, Pleopeltis mexicana, Polypodium plebeium, P. rhodopleuron, Ponthieva ephippium, Rhynchostele rossii y Tillandsia imperialis.

Las plantas herbáceas son abundantes y se encuentra gran diversidad de ellas en este bosque; entre ellas se registran: Arisaema dracontium, Arracacia tolucensis, Ascyrum hypericoides, Aspidotis meifolia, Asplenium auriculatum, A. monanthes, A. praemorsum, Begonia gracilis, Blechnum glandulosum, Calanthe calanthoides, Campyloneurum angustifolium, Cheilanthes notholaenoides, Crusea longiflora, Cuphea calaminthifolia, C. cyanea, Cystopteris fragilis, Chimaphila maculata, Dennstaedtia distenta, Dryopteris wallichiana, Duchesnea indica, Echeveria rosea, Fuchsia encliandra, Llavea cordifolia, Malaxis brachyrhynchos, M. carnosa, M. excavata, Mitchella repens, Nertera granadensis, Orthrosanthus exsertus, Penstemon barbatus, Phanerophlebia nobilis, Pinguicula moranensis, Pleopeltis crassinervata, Polypodium plebeium, $P$. plesiosorum, P. puberulum, Ponthieva ephippium, Pteris cretica, Priva mexicana, Sedum retusum, Selaginella extensa, Senecio picridis y Spigelia longiflora.

La Joya del Hielo ocupa una pequeña depresión en la parte alta de los cerros. Es un área única en el estado, por la presencia de dos especies de Magnolia y por su riqueza florística, por lo que ha sido considerada como una de las áreas núcleo de la Reserva de la Biosfera Sierra Gorda. La presencia de Cupressus lusitanica, Carpinus caroliniana y Ostrya virginiana en el bosque mesófilo refleja la influencia del clima más fresco de la parte alta de las montañas y varias de las especies arbustivas y herbáceas encontradas aquí se comparten con los bosques de pino-encino de la región. En condiciones más secas, este bosque es sustituido por el bosque de Pinus greggii y Quercus polymorpha, como se aprecia en los alrededores de El Lobo y El Madroño.

En el Llano Chiquito, por arriba de los $2000 \mathrm{~m}$ de altitud, sobre laderas calizas cársticas, se desarrolla un bosque parecido al anterior, con dominancia de Cupressus lusitanica.

Composición florística. Se ha conjuntado una lista florística de las plantas vasculares del área, compuesta por 130 familias, 465 géneros y 774 especies (apéndice 1); el grupo de plantas vasculares más numeroso es el de las angiospermas, seguido de las pteridofitas y grupos afines; las gimnospermas están representadas por pocas especies. Las dicotiledóneas son las mejor representadas (casi el 75\%), tanto a nivel de familia, como de género y especie (cuadro 1).

Cuadro 1. Abundancia de los grupos de plantas vasculares.

\begin{tabular}{|c|c|c|c|}
\hline Grupo & $\begin{array}{l}\text { Número de familias } \\
\text { y porcentaje }\end{array}$ & $\begin{array}{l}\text { Número de géneros } \\
\text { y porcentaje }\end{array}$ & $\begin{array}{l}\text { Número de especies } \\
\text { y porcentaje }\end{array}$ \\
\hline Pteridophyta y afines & $15(11.5 \%)$ & $43(9.2 \%)$ & $96(12.4 \%)$ \\
\hline Gymnospermae & $3(2.3 \%)$ & $3(0.6 \%)$ & $3(0.4 \%)$ \\
\hline Monocotyledonae & $15(11.5 \%)$ & $64(13.8 \%)$ & $99(12.8 \%)$ \\
\hline Dicotyledonae & $97(74.6 \%)$ & 355 (76.3\%) & $576(74.4 \%)$ \\
\hline TOTAL & 130 & 465 & 774 \\
\hline
\end{tabular}


Las familias más diversas en cuanto a número de géneros son Compositae con 47 (10.11\%), Leguminosae con 33 (7.10\%), Orchidaceae con 25 (5.38\%), Rubiaceae con 16 (3.44\%), Gramineae con $13(2.80 \%)$, Aspleniaceae y Euphorbiaceae con 11 (2.37\%), Malvaceae, Scrophulariaceae y Verbenaceae con 9 (1.94\%), y Adiantaceae, Labiatae y Solanaceae con 8 (1.72\%); el resto de las familias comprenden 258 géneros que representan el $55.48 \%$ (figura $4 a$ ).

Las familias más abundantes en cuanto a número de especies son Compositae con 74 (9.56\%), Leguminosae con 56 (7.24\%), Orchidaceae con $36(4.65 \%)$, Solanaceae con 33 (4.26\%), Rubiaceae y Polypodiaceae con 23 (2.97\%), Verbenaceae con 22 (2.84\%), Aspleniaceae con $21(2.71 \%)$,
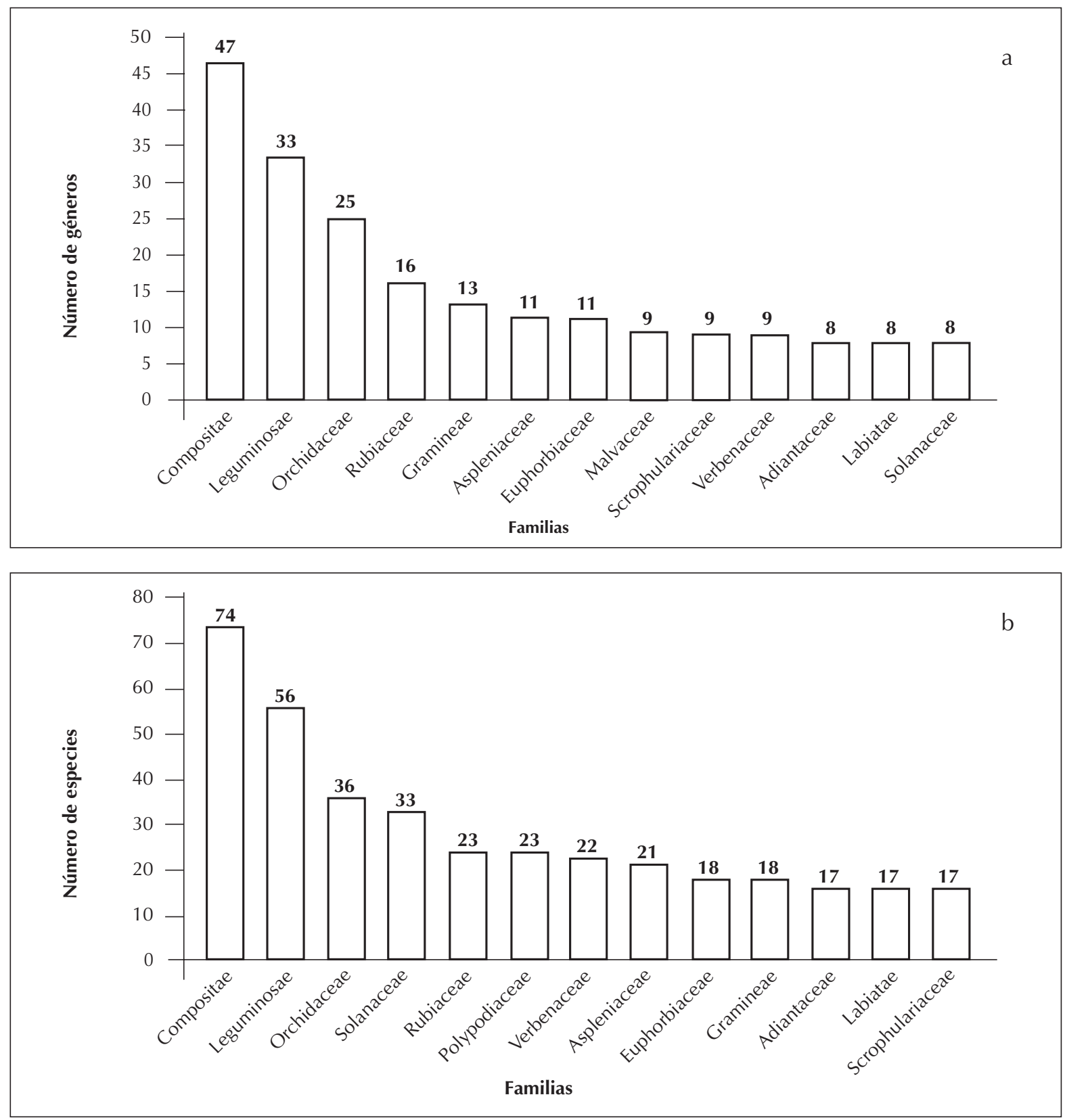

Figura 4. a) Abundancia de géneros por familia; los 258 géneros restantes pertenecen a familias varias. b) Abundancia de especies por familia; las 400 especies restantes pertenecen a diversas familias. 
Euphorbiaceae y Gramineae con 18 (2.33\%) y Adiantaceae y Labiatae con 17 (2.20\%); las familias restantes incluyen 400 especies que representan el $51.68 \%$ (figura $4 \mathrm{~b}$ ).

Con respecto al número de especies por forma de vida, las hierbas son las más abundantes con 384 (49.6\%), seguidas por los arbustos con 151 (19.5\%), árboles con 121 (15.6\%), bejucos con $63(8.1 \%)$, epífitas con $47(6.1 \%)$ y parásitas con $8(1.0 \%)$ (figura $5 a)$.
Las familias mejor representadas por formas de vida en el área de estudio son: a) hierbas: Compositae (39 especies), Orchidaceae (25), Aspleniaceae (21), Gramineae (18), Labiatae (17), Leguminosae (16), Adiantaceae (15), Solanaceae (13) y Verbenaceae (12); b) arbustos: Compositae (33 especies), Solanaceae y Leguminosae (14), Rubiaceae (11) y Verbenaceae (8); c) árboles: Leguminosae (16 especies), Lauraceae (12), Fagaceae (11) y Myrtaceae (5); d) epífitas:
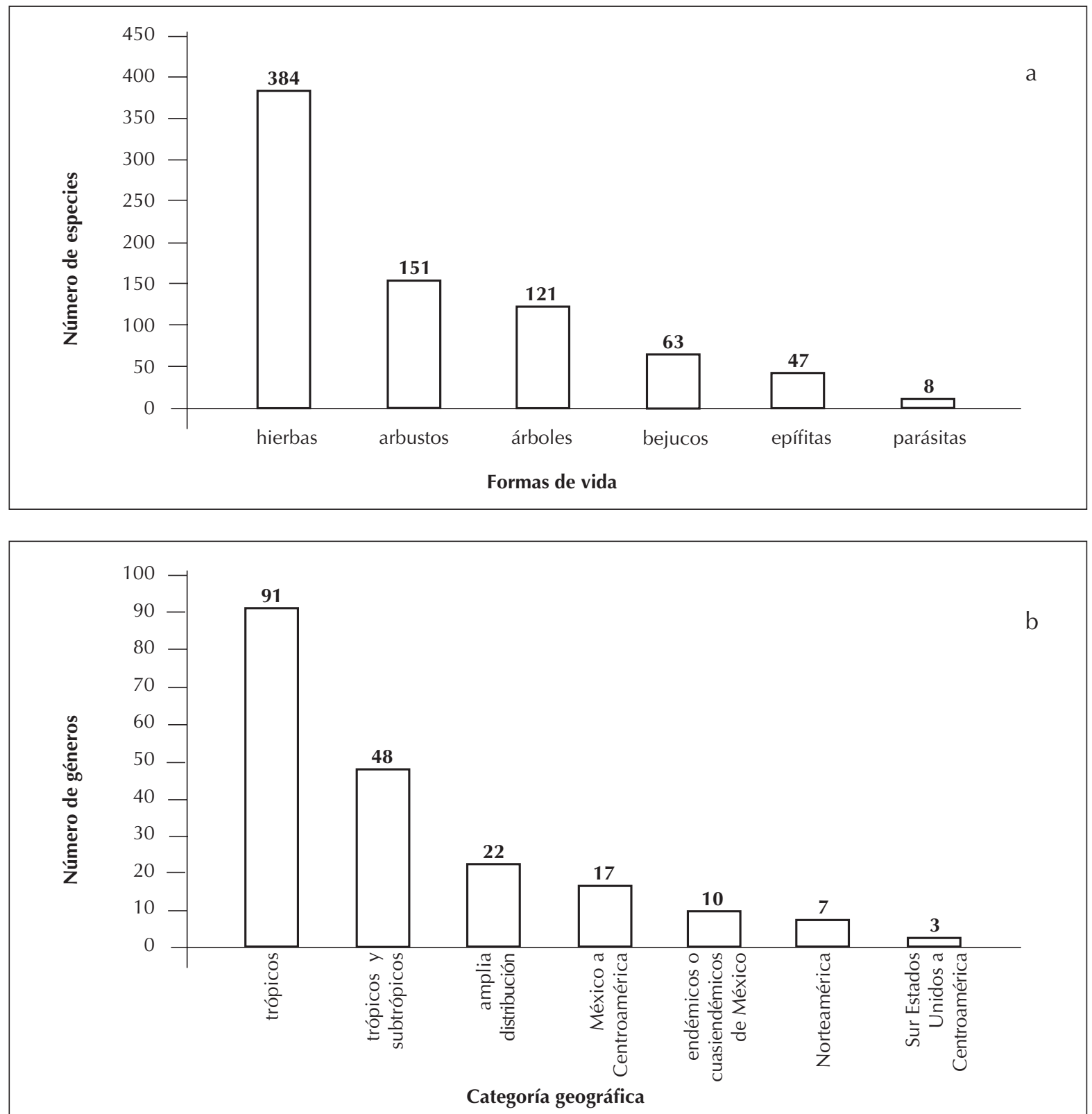

Figura 5. a) Número de especies por forma de vida. b) Distribución de los géneros presentes en Landa de Matamoros en el continente americano. 
Polypodiaceae (19 especies), Orchidaceae (11) y Bromeliaceae (5); e) bejucos: Leguminosae (10 especies), Asclepiadaceae (7), Liliaceae y Vitaceae (6), y f) parásitas: Loranthaceae (6 especies).

Análisis de distribución a nivel de género. Los 465 géneros registrados en el área se agruparon en seis categorías de acuerdo con su distribución actual: 198 (42.6\%) se distribuyen exclusivamente en América, 109 (23.4\%) en las zonas tropicales y subtropicales de todo el mundo, $56(12.0 \%)$ en los trópicos, 51 (11.0\%) son de amplia distribución, 42 (9.0\%) se restringen al Hemisferio Norte y $9(1.9 \%)$ a zonas templadas y subtropicales.

La mayoría de los géneros del área de estudio restringen su distribución al continente americano; de ellos, 91 se distribuyen de manera exclusiva en las regiones tropicales, seguidos de los géneros con distribución en los trópicos y subtrópicos (48), de amplia distribución (22), de México a América Central (17), endémicos o cuasi-endémicos de México (10), solamente en América del Norte (7) y del sur de Estados Unidos a América Central (3) (figura 5b).

Análisis de las formas de vida a nivel de género. De los 465 géneros recolectados en los bosques mesófilos de Landa de Matamoros, 229 son hierbas, 88 arbustos, 77 árboles, 41 bejucos, 26 epífitas y 4 parásitas.

Los géneros arbóreos más abundantes son los que se distribuyen en América (20), en los trópicos y subtrópicos y en el Hemisferio Norte (21 en cada caso); de los géneros arbustivos, 47 se restringen al continente americano, 19 son tropicales y 15 tropicales y subtropicales; la mayoría de los géneros herbáceos se distribuyen en América (95), o son de distribución tropical y subtropical (59) y de amplia distribución (39); los bejucos se distribuyen principalmente en América (16), en los trópicos y subtrópicos (11) y en los trópicos (8); gran parte de las epífitas (16) se distribuyen en América, al igual que las parásitas (4) (apéndice 1).

\section{Discusión y Conclusiones}

La lista florística preliminar del bosque mesófilo de montaña del municipio de Landa de Matamoros, Querétaro, comprende 774 especies, incluidas en 465 géneros y 130 familias, que representan el 25\% de la riqueza florística estimada para este tipo de vegetación en México (Rzedowski, 1991), lo que revela la alta diversidad biológica concentrada en la zona de estudio, a pesar del área tan reducida que ocupa. La gran riqueza florística de esta zona se puede explicar por la coincidencia de varios factores, entre los que se encuentran: a) la compleja historia geológica y biogeográfica del área (Luna et al., 1999, 2001); b) el amplio gradiente altitudinal en que se distribuye el bosque; c) la accidentada topografía, principalmente la existencia de laderas escarpadas y cañadas húmedas; d) la complejidad de condiciones climáticas; e) el ecotono frecuente con otros tipos de vegetación (bosque tropical subcaducifolio, bosque de pino y bosque de encino); y f) el alto grado de perturbación que permite el establecimiento de plantas arvenses o ruderales (Zamudio y Fernández, 1990).

En Landa de Matamoros, al igual que en otros bosques mesófilos mexicanos, la estructura y composición florística del bosque cambia de un sitio a otro en función de varios factores como el gradiente altitudinal, la complejidad climática, las diferencias ocasionadas por la exposición y el grado de disturbio, entre otros (Zamudio et al., 1992). El crecimiento frondoso de musgos y selaginelas sobre el suelo, los troncos y las ramas de los árboles refleja la abundancia de humedad en el ambiente (Zamudio y Fernández, 1990; Zamudio et al., 1992).

De las familias que Rzedowski (1996) menciona que prosperan preferentemente en esta comunidad, en el extremo oriental del municipio de Landa de Matamoros se presentan las siguientes: Aquifoliaceae, Balanophoraceae, Begoniaceae, Clethraceae, Lauraceae, Magnoliaceae, Myrsinaceae, Orchidaceae, Piperaceae, Selaginellaceae, Styracaceae y Theaceae.

En la lista florística se puede apreciar que en el área estudiada prevalecen las plantas herbáceas (49.6\%), siguiendo en orden decreciente las formas de vida arbustiva (19.5\%), arbórea (15.6\%), los bejucos (8.1\%) y las epífitas (6.1\%). La mayor abundancia de hierbas se podría explicar en parte por el alto grado de perturbación y la alta humedad atmosférica.

Muchas de las especies registradas en el área son endémicas de México y algunas restringen su distribución a la Sierra Madre Oriental. Entre las endémicas de México se encuentran: Bouvardia xylosteoides, Carya ovata var. mexicana, Cuphea calaminthifolia, C. lanceolata, C. salicifolia, Encyclia mariae, Epidendrum longipetalum, Eugenia xalapensis, Isochilus unilateralis, Lycaste consobrina, Magnolia schiedeana, Ocotea klotzschiana, O. psychotrioides, Pavonia uniflora, Phymosia umbellata, Pitcairnia ringens, Rhamnus capraefolia, Robinsonella discolor, Solanum iopetalum, Stanhopea tigrina, Tilia mexicana, Tillandsia deppeana, Vernonia alamanii y Witheringia stellata; algunas de las especies endémicas de la Sierra Madre Oriental son: Agave celsii, Annona globiflora, Carya palmeri, Cinnamomum bractefoliaceum, C. effusum, Clethra kenoyeri, C. pringlei, Dichaea neglecta, Ilex condensata, Inga huastecana, Lobelia orientalis, Panicum xalapense, Physalis queretaroensis, Senna racemosa var. moctezumae, Ternstroemia sylvatica y Vernonia arctioides, entre otras.

En la Norma Oficial Mexicana (Diario de la Federación, 2001) se cita a Bouvardia xylostioides y Ostrya virginiana dentro de la categoría de especies raras; a Campyloneurum phyllitidis, Carpinus caroliniana, Magnolia schiedeana y Stanhopea tigrina dentro de la categoría de amenazadas; a Cupressus lusitanica como sujetas a protección especial y a Litsea glaucescens, Magnolia dealbata y Tilia mexicana como especies en peligro de extinción. Muchas de ellas son 
endémicas, como en el caso de Bouvardia xylosteoides, Encyclia mariae, Magnolia schiedeana, Magnolia dealbata, Stanhopea tigrina y Tilia mexicana, por lo que urge tomar medidas para su protección.

A pesar de la riqueza florística de este bosque en Querétaro, es notoria la ausencia de Acer skutchii, Fagus mexicana, Nyssa sylvatica, Podocarpus reichei y Weinmannia pinnata, especies que se presentan en los estados vecinos de Hidalgo y San Luis Potosí (Luna et al., 1994). La ausencia de estos taxones y las diferencias en la composición florística con los bosques aledaños del sur dentro de la misma Sierra Madre Oriental (v.gr. Tlanchinol, Huayacocotla, Helechales, Tenango de Doria, Zacualtipán, Eloxochitlán, entre varios otros), apoyan la idea de que esta sierra no representa una unidad natural, sino que más bien puede dividirse en dos porciones, una sur y otra norte, que están separadas entre sí por la cuenca del Pánuco (Luna et al., 1999).

La mayoría de los géneros presentes en el área se distribuyen exclusivamente en América (47\%), lo cual concuerda con lo que propone Rzedowski (1996), quien afirma que aproximadamente el $46 \%$ de los géneros de fanerógamas del bosque mesófilo de montaña presentan una distribución que va de México hasta América del Sur, seguidos de los que se distribuyen en los trópicos y subtrópicos (23.4\%).

En la actualidad, en el área de estudio el bosque mesófilo de montaña se encuentra muy fragmentado y perturbado. Las principales causas que ocasionan su destrucción están ligadas estrechamente a las actividades productivas de los habitantes de la región. Tradicionalmente estos bosques se utilizaban para establecer plantaciones de café, en las que el estrato arbóreo se conservaba como sombra; sin embargo, en los últimos años extensas superficies de bosque han sido desmontadas para incrementar los potreros y las áreas dedicadas a la agricultura. También ha sido importante en diferentes épocas la extracción forestal del liquidámbar para la fabricación de palillos y del palo escrito para la elaboración de guitarras, muebles y artesanías (Zamudio y Fernández, 1990).

A pesar de que el municipio de Landa de Matamoros se encuentra dentro de la Reserva de la Biosfera Sierra Gorda, decretada en 1997, la dinámica de destrucción del bosque mesófilo de montaña y de otras comunidades vegetales es continua y existe el riesgo de que en un mediano plazo la mayor parte de las áreas con bosque mesófilo de montaña desaparezcan de la zona. Algunas localidades como La Joya del Hielo y el Llano Chiquito han sido consideradas como áreas núcleo de la reserva; no obstante, aún no se observa una política clara para su manejo y conservación por parte de los grupos que administran dicha reserva.

Los pobladores de las comunidades establecidas en las zonas de bosque mesófilo como Agua Zarca, Camarones, El Rincón, Jagüey Colorado y La Florida, entre otras, están concientes del daño causado a la vegetación, sobre todo porque ellos mismos empiezan a notar la ausencia de ciertas especies animales y vegetales que antes eran comunes y que utilizaban para cubrir sus necesidades.

Es importante que las instituciones que están a cargo de la reserva pongan en marcha estrategias serias para el manejo de la reserva, que además de dar protección a las áreas mejor conservadas fomenten la investigación ecológica, con énfasis en la regeneración y restauración de la vegetación y en la búsqueda de formas alternativas de producción, que permitan obtener ingresos a los pobladores sin destruir el bosque.

Es necesario además, fomentar una cultura ecológica que incluya a los pobladores de la región, para que sean ellos quienes se hagan cargo de la protección de sus recursos naturales. En relación con este aspecto, la educación juega un papel importante en la búsqueda de soluciones a los grandes problemas ecológicos que ahora nos aquejan.

\section{Agradecimientos}

Se agradece a Raúl Contreras, Carlos Ruiz y dos árbitros anónimos por la revisión del manuscrito. A Carlos Ruiz por su ayuda de campo y la elaboración de los mapas. A Engracia Hernández por su ayuda para la determinación del clima. A las siguientes personas su ayuda en la determinación del material: Enrique Ortiz (Compositae), Susana Valencia (Fagaceae), Marta Martínez (Euphorbiaceae), Nelly Diego (Cyperaceae), Francisco Lorea (Lauraceae), Ramiro Cruz (Leguminosae), Miguel Ángel Soto (Orchidaceae) y Susana Peralta (Verbenaceae). A los proyectos DGAPA-PAPIIT IN205799, CONACYT 31879-N e Instituto de Ecología, A.C., cuenta 902-03.

\section{Literatura citada}

Anónimo. 1981. Atlas Nacional del Medio Físico. Secretaría de Programación y Presupuesto. México, D.F.

Argüelles E., Fernández R. y Zamudio S. 1991. Listado florístico preliminar del estado de Querétaro. Flora del Bajío y de Regiones Adyacentes. Fascículo Complementario II:1-155.

Arreguín S.M.L., Cabrera L.G., Fernández N.R., Orozco L.C., Rodríguez C.B. y Yépez B.M. 1997. Introducción a la flora del estado de Querétaro. CONCYTEQ. Querétaro, México.

Brummitt R.K. y Powell C.E. (Eds.) 1992. Authors of plant names. Royal Botanic Gardens. Kew.

CONABIO-Estadigrafía. 1997. Carta de climas México. Sistema de Köppen modificado por E. García. Escala 1:1,000,000. México, D.F.

Consejo de Recursos Minerales. 1992. Monografía geológicominera del estado de Querétaro. Consejo de Recursos Minerales. Pachuca, México.

Diario Oficial de la Federación. 1997. Decreto de la Reserva de la Biosfera de la Sierra Gorda, Tercera sección, SEMARNAP (Secretaría de Medio Ambiente, Recursos Naturales y Pesca), pp. 1-11, 19 de mayo de 1997.

Diario Oficial de la Federación. 2001. Protección ambiental-especies nativas de México de flora y fauna silvestres-categorías de riesgo 
y especificaciones para su inclusion, exclusión o cambio-lista de especies en riesgo, Norma Oficial Mexicana NOM-059ECOL-2001, 6 marzo de 2002.

Díaz-Barriga H. y Palacios-Ríos M. 1992. Lista preliminar de especies de pteridofitas de los estados de Guanajuato, Michoacán y Querétaro. Flora del Bajío y de Regiones Adyacentes. Fascículo Complementario III:1-57.

Engler A. y Diels L. 1936. Syllabus der Pflanzenfamilien. 11a. ed., Borntraeger. Berlín.

Flores O. y Gerez P. 1988. Conservación en México: Síntesis sobre Vertebrados Terrestres, Vegetación y Uso del Suelo. Instituto Nacional de Investigaciones sobre Recursos Bióticos y Conservación Internacional. México, D.F.

García E. 1988. Modificaciones al Sistema de Clasificación Climática de Köppen. Editado por la autora. México, D.F.

Halffter G. 1992. Diversidad biológica y cambio global. Ciencia y Desarrollo 18:33-38.

INEGI (Instituto Nacional de Estadística Geografía e Informática). 1981. Carta topográfica Ahuacatlán, F14C39, 1:50,000. México, D.F.

INEGI (Instituto Nacional de Estadística Geografía e Informática). 1985. Carta de uso de suelo y vegetación. Ciudad Valles F14-8. 1:250,000. México, D.F.

INEGI (Instituto Nacional de Estadística Geografía e Informática). 1992. Síntesis geográfica del estado de Querétaro. INEGI. México, D.F

INEGI (Instituto Nacional de Estadística Geografía e Informática). 1995a. Carta topográfica Jacala, F14C49, 1:50,000, México, D.F.

INEGI (Instituto Nacional de Estadística Geografía e Informática). 1995b. Fotografías aéreas del estado de Querétaro. 1:75,000.

International Institute for Aerospace Survey and Earth Sciences. 1997. ILWIS 2.1. The Integrated Land Information System. Enschede, Holanda.

Lot A. y Chiang F. 1986. Manual de Herbario. Consejo Nacional de la Flora México. México, D.F.

Luna I., Almeida L., Villers L. y Lorenzo L. 1988. Reconocimiento florístico y consideraciones fitogeográficas del bosque mesófilo de montaña de Teocelo, Veracruz. Boletín de la Sociedad Botánica de México 48:35-63.

Luna I., Almeida L. y Llorente J. 1989. Florística y aspectos fitogeográficos del bosque mesófilo de montaña de las cañadas de Ocuilan, estados de Morelos y México. Anales del Instituto de Biología, Universidad Nacional Autónoma de México, Serie Botánica 59:63-87.
Luna I., Ocegueda S. y Alcántara O. 1994. Florística y notas biogeográficas del bosque mesófilo de montaña del municipio de Tlanchinol, Hidalgo, México. Anales del Instituto de Biología, Universidad Nacional Autónoma de México, Serie Botánica 65:31-62.

Luna I., Alcántara O., Espinosa D. y Morrone J.J. 1999. Historical relationships of the Mexican cloud forests: A preliminary approach applying Parsimony Analysis of Endemicity. Journal of Biogeography 26:1299-1306.

Luna I., Morrone J.J., Alcántara O. y Espinosa D. 2001. Biogeographical affinities among Neotropical cloud forests. Plant Systematics and Evolution 228:229-239.

Mabberley D.J. 1997. The Plant-Book. A portable dictionary of the vascular plants. Cambridge University Press. Cambridge.

Mickel J. y Beitel J.M. 1988. Pteridophyte flora of Oaxaca, Mexico. Memoirs of the New York Botanical Garden 46:1-566.

Mueller-Dombois D., Little M. y van der Hammen T. 1988. Manual of methods for transect studies. Comparative Studies of Tropical Mountain Ecosystems. International Union of Biological Sciences. Lausana.

Puig H. 1976. Végétation de la Huasteca, Mexique. Mision Archéologique et Ethonologique Française au Mexique. México. D.F.

Rzedowski J. 1978. Vegetación de México. Limusa. México, D.F.

Rzedowski J. 1991. Diversidad y orígenes de la flora fanerogámica de México. Acta Botanica Mexicana 14:3-21.

Rzedowski J. 1996. Análisis preliminar de la flora vascular de los bosques mesófilos de montaña de México. Acta Botanica Mexicana 35:25-44.

Secretaría de Comunicaciones y Transportes. 1986. Mapa de carreteras, estado de Querétaro, México 1: 400,000. México, D.F.

Toledo V.M. 1988. La diversidad biológica de México. Ciencia y Desarrollo 81:17-30.

Zamudio S. 1984. La vegetación de la cuenca del río Estórax en el estado de Querétaro y sus relaciones fitogeográficas. Tesis de licenciatura. Facultad de Ciencias, Universidad Nacional Autónoma de México, México, D.F., 275 pp.

Zamudio S. y Fernández R. 1990. El bosque mesófilo de montaña en el estado de Querétaro. En: Libro de Resúmenes, XI Congreso Mexicano de Botánica. pp. 365. Oaxtepec, Morelos, México.

Zamudio S., Rzedowski J., Carranza G.E. y Calderón de Rzedowski G. 1992. La Vegetación en el estado de Querétaro. Instituto de Ecología. Centro Regional del Bajío. Pátzcuaro, Michoacán, México. 
Apéndice 1. Lista florística del municipio de Landa de Matamoros, Querétaro

Las abreviaturas que anteceden al nombre científico corresponden a la forma de vida predominante: $\mathrm{A}=\mathrm{a}$ rbol, $\mathrm{Ar}=$ arbusto, $\mathrm{H}=$ hierba, $\mathrm{E}=$ epífita, $\mathrm{B}=$ bejuco, $\mathrm{P}=$ parásita. Las abreviaturas que preceden al nombre científico corresponden al tipo de distribución actual del género: $\mathrm{Am}=$ restringido al continente americano; $\mathrm{TrSub}=$ tropical y subtropical; $\mathrm{Cos}=$ de amplia distribución; $\operatorname{Tr}=$ tropical; $\mathrm{HN}=$ Hemisferio Norte y $\mathrm{TmSb}=$ templado subtropical). Por último, se incluye en algunos casos datos relativos a la distribución americana de algunos géneros: 1 = endémico de México; 2 = restringido a Mesomérica; 3 = trópicos de América.

\section{PTERIDOPHYTA Y GRUPOS AFINES}

\section{ADIANTACEAE}

H. Adiantum concinnum Humb. et Bonpl. ex Willd.

Cos

H. Adiantum tenerum Sw.

$\mathrm{H}$. Adiantum trapeziforme L.

H. Bommeria pedata (Sw.) Fourn. Am

E. Cheilanthes aemula Maxon

H. Cheilanthes notholaenoides (Desv.) Maxon ex Weath.

Cos

$\mathrm{H}$. Hemionitis palmata L.

H. Llavea cordifolia Lag.

H. Mildella intramarginallis (Kaulf. ex Link) Trevis

H. Pityrogramma calomelanos (L.) Link

Am

H. Pityrogramma ebenea (L.) Proctor

H. Pteris cretica L.

Am 2

$\mathrm{Tr}$

H. Pteris grandifolia L.

H. Pteris longifolia L.

H. Pteris orizabae Martens et Galeotti

H. Pteris pulchra Schltdl. et Cham.

E. Pteris quadriaurita Retz.

\section{ASPLENIACEAE}

H. Asplenium auriculatum Sw.

Cos

H. Asplenium cuspidatum Lam.

$\mathrm{H}$. Asplenium monanthes L.

H. Asplenium praemorsum Sw.

H. Asplenium sessilifolium Desv.

H. Asplenium sphaerosphorum A.R.Sm.

$\mathrm{H}$. Asplenium vesiliensis Kunze

H. Ctenitis nigrovenia (Christ) Copel

$\operatorname{Tr}$

H. Ctenitis submarginalis (Langsd. et Fisch.) Ching

H. Cystopteris fragilis (L.) Bernh.

TmSub

H. Cystopteris membranifolia Mickel

H. Diplazium franconis Liebm.

TrSub

H. Dryopteris wallichiana (Spreng.) Hyl.

H. Elaphoglossum glaucum Moore

H. Holodictyum ghiesbreghtii (Fourn.) Maxon

H. Megalastrum pulverulentum (Poir.) A.R.Sm. et R.C.Moran

H. Phanerophlebia nobilis (Schltdl. et Cham.) Presl

$\operatorname{Cos}$

H. Phanerophlebia remotispora Fourn.

H. Schaffneria nigripes Fée

H. Tectaria heracleifolia (Willd.) Underw.

H. Tectaria transiens (Morton) A.R.Sm.

Cos

TrSub

Am

Am

TrSub

BLECHNACEAE

H. Blechnum glandulosum Kaulf. in Link

Cos

CYATHEACEAE

A. Cyathea firma Mett. ex Jun

TrSub 


\section{DENNSTAEDTIACEAE}

H. Dennstaedtia bipinnata (Cav.) Maxon

TrSub

H. Dennstaedtia cicutaria (Sw.) Moore

H. Dennstaedtia distenta (Kunze) Moore

Ar. Dennstaedtia globulifera (Poir.) Hieron.

H. Pteridium caudatum (L.) Maxon

Cos

EQUISETACEAE

H. Equisetum myriochaetum Schltdl. et Cham.

Cos

HYMENOPHYLLACEAE

E. Hymenophyllum tunbrigense (L.) J.Sm. Cos

E. Trichomanes radicans Sw. TrSub

\section{LOPHOSORIACEAE}

H. Lophosoria quadripinnata (J.F.Gmel.) C.Chr.

Am

\section{LYCOPODIACEAE}

H. Lycopodium clavatum L.

MARATTIACEAE

H. Marattia weinmanniifolia Liebm.

$\operatorname{Cos}$

\section{OPHIOGLOSSACEAE}

H. Botrychium decompositum Martens et Galeotti Cos

H. Ophioglossum crotalophoroides Walter

$\operatorname{Cos}$

H. Ophioglossum reticulatum L.

\section{POLYPODIACEAE}

H. Aspidotis meifolia (D.C. Eaton) Pic.Serm.

$\mathrm{HN}$

H. Campyloneurum angustifolium (Sw.) Fée

$\mathrm{Am}$

E. Campyloneurum xalapense Fée

E. Campyloneurum phyllitidis (L.) Presl

H. Holodyctium ghiesbreghtii (Fourn.) Maxon

Am

E. Pecluma cyathicola Copel.

$\mathrm{Am}$

1

E. Pecluma plumula (Humb. et Bonpl. ex Willd.) Price

E. Pecluma ptilodom (Kunze) Price var. bourgaeana (E.Fourn.) A.R.Sm.

E. Phlebodium areolatum (Humb. et Bonpl.ex Willd.) J.Sm.

E. Pleopeltis crassinervata (Fée) Moore

E. Pleopeltis fallax (Schltdl. et Cham.) Mickel et Beitel

E. Pleopeltis interjecta (Weath.) Mickel et Beitel

E. Pleopeltis mexicana (Feé) Mickel et Beitel

E. Polypodium ferruginea (Martens et Galeotti) Mickel

E. Polypodium fraternum Schltdl. et Cham.

E. Polypodium furfuraceum Schltdl. et Cham.

E. Polypodium longepinnulatum Fourn.

E. Polypodium plebeium Schltdl. et Cham.

E. Polypodium plesiosorum Kuntze

E. Polypodium polypodioides (L.) Watt.

E. Polypodium polypodioides (L.) Watt. var. aciculare Weath.

H. Polypodium puberulum Schltdl. et Cham.

E. Polypodium rhodopleuron Kunze

\section{SCHIZAEACEAE}

H. Anemia mexicana Klotzsch var. mexicana

TrSub

H. Anemia phyllitidis (L.) Sw.

H. Lygodium venustum Sw. 
SELAGINELLACEAE

H. Selaginella delicatissima Lind. ex A.Braun

TrSub

H. Selaginella extensa Underw.

H. Selaginella harrisii Underw. et Hieron.

H. Selaginella reflexa Underw.

H. Selaginella stenophylla A.Braun

\section{THELYPTERIDACEAE}

H. Thelypteris blanda (Fée) C.F. Reed

TmSub

H. Thelypteris dentata (Forssk.) E.St. John

H. Thelypteris linkiana (C. Presl) R. Tryon

H. Thelypteris oligocarpa (Humb. et Bonpl. ex Willd.) Ching

H. Thelypteris ovata E.St. John var. lindheimeri (C. Chr.) E.St. John

H. Thelypteris patens (Sw.) Small var. patens

H. Thelypteris pilosa (Martens et Galeotti) Crawford

H. Thelypteris schaffneri (Fée) C.F.Reed

$\mathrm{H}$. Thelypteris tetragona (Sw.) Small

H. Thelypteris torresiana (Gaudich.) Alston

E. Vittaria graminifolia Kaulfuss

TrSub

\section{GYMNOSPERMAE}

CUPRESSACEAE

A.Cupressus lusitanica Kunth

PINACEAE

A. Pinus greggii Engelm. ex Parl.

$\mathrm{HN}$

\section{TAXACEAE}

A. Taxus globosa Schltdl.

$\mathrm{HN}$

\section{ANGIOSPERMAE}

\section{MONOCOTYLEDONAE}

\section{AGAVACEAE}

H. Agave celsii Hook.

\section{AMARYLLIDACEAE}

B. Bomarea edulis (Tussac) Herb.

$\mathrm{Am}$

B. Bomarea hirtella (Kunth) Herb.

B. Bomarea sp.

H. Zephyranthes brevipes (Baker ex Donn.Sm.) Standl.

Am

H. Zephyranthes lindleyana Herb.

\section{ARACEAE}

H. Arisaema dracontium (L.) Schott.

TrSub

\section{BROMELIACEAE}

E. Guzmania scherzeriana Mez var. guatemalensis (L.B.Sm.)

L.B.Sm.

H. Pitcairnia ringens Klotzsch ex Link, Klotzsch et Otto

E. Tillandsia bartramii Elliott

E. Tillandsia deppeana Steud.

E. Tillandsia imperialis E. Marren ex Mez

E. Tillandsia polystachia (L.) L.

E. Tillandsia sp. 


\section{CANNACEAE}

H. Canna indica L.

$\mathrm{Am}$

H. Canna sp.

\section{COMMELINACEAE}

H. Campelia zanonia (L.) Kunth

H. Commelina tuberosa L.

H. Gibasis geniculata (Jacq.) Rohweder

H. Gibasis pellucida (M.Martens et Galeotti) Hunt

H. Tripogandra serrulata (Vahl) Handlos

Am

$\mathrm{Am}$

\section{CYPERACEAE}

H. Cyperus hermaphroditus (Jacq.) Standl.

TrSub

H. Cyperus uniflorus Torr. et Hook.

H. Cyperus sp.

H. Scleria oligantha Michx.

H. Scleria sp.

TrSub

\section{DIOSCOREACEAE}

B. Dioscorea convolvulacea Schltdl. et Cham.

TrSub

\section{GRAMINEAE}

H. Agrostis sp.

H. Arundinella deppeana Nees ex Steud.

Cos

H. Arundinella sp.

H. Digitaria insularis (L.) Mez

H. Gouinia virgata (J. Presl) Scribn.

H. Lasiacis nigra Davidse

H. Leersia sp.

H. Oplismenus compositus (L.) P.Beauv.

H. Panicum pilosum Sw.

H. Panicum sp.

H. Panicum xalapense Kunth

H. Paspalum conjugatum Bergius

TrSub

H. Paspalum notatum P.Beauv.

H. Paspalum sp.

H. Pereilema crinitum J. Presl

H. Rhynchelytrum repens (Willd.) C.E.Hubb.

H. Sporobolus indicus (L.) R.Br.

H. Zeugites sp.

TrSub

Am

$\operatorname{Tr}$

TrSub

Am

TrSub

TrSub

IRIDACEAE

H. Sisyrinchium angustissimum (B.L.Rob. et Greenm.) Greenm. et C.H.Thomps.

H. Tigridia pavonia (L.f.) DC.

H. Orthrosanthus exsertus (R.C.Foster) Ravenna

Am

$\mathrm{Tr}$

$\operatorname{Tr}$

Am

\section{LILIACEAE}

H. Echeandia mexicana Cruden

H. Hypoxis decumbens L.

H. Nothoscordum bivalve (L.) Britton

Am

B. Smilax aristolochiifolia Mill.

Am

B. Smilax bona-nox L.

B. Smilax domingensis Willd.

B. Smilax glauca Walter

B. Smilax jalapensis Schltdl.

B. Smilax mollis Humb. et Bonpl. ex Willd.

Am

TrSub

Am

TrSub 
MARANTACEAE

H. Maranta arundinacea L.

MUSACEAE

H. Heliconia schiedeana Klotzsch

\section{ORCHIDACEAE}

H. Beloglottis costaricensis (Rchb.f.) Schltdl.

H. Bletia purpurea (Lam.) DC.

H. Calanthe calanthoides (A. Rich et Galeotti) Hamer et Garay

E. Catasetum integerrimum Hook.

H. Cranichis mexicana (A.Rich. et Galeotti) Schltr.

H. Cranichis subumbelata A.Rich. et Galeotti

H. Cranichis sylvatica A.Rich. et Galeotti

H. Cyclopogon luteo-albus (A.Rich. et Galeotti) Schltr.

H. Dichaea neglecta Schltr.

H. Encyclia cochleata (L.) Lem.

H. Encyclia mariae (Ames) Hoehne

H. Epidendrum longipetalum A.Rich. et Galeotti

H. Epidendrum propinquum A.Rich. et Galeotti

H. Habenaria sp.

E. Isochilus unilateralis B.L.Rob.

H. Laelia anceps Lindl.

H. Lepanthes papilionacea Salazar, Soto Arenas et O. Suárez

E. Lycaste consobrina Rchb.f.

H. Malaxis brachyrrhynchos (Rchb.f.) Ames

H. Malaxis carnosa (Kunth) C.Schweinf.

H. Malaxis excavata (Lindl.) O. Kuntze

H. Malaxis histionantha (Link Kl. et Otto) Garay et Dunsteru.

H. Malaxis sp.

E. Maxillaria densa Lindl.

H. Maxillaria variabilis Bateman ex Lindl.

E. Nidema boothii (Lindl.) Schltr.

E. Oncidium sphacelatum Lindl.

E. Pleurothallis nicaraguensis Rchb.f.

H. Ponthieva ephippium Rchb.f.

H. Ponthieva racemosa (Walter) Mohr

E. Rhynchostele rossii (Lindl.) Soto Arenas et Salazar

H. Sarcoglottis schaffneri (Rchb.f.) Ames

H. Spiranthes adnata (Sw.) Benth. ex Fawc.

H. Spiranthes costaricensis Rchb.f.

E. Stanhopea tigrina Bateman et Lindl.

E. Trichocentrum candidum Lindl.

PALMAE

H. Brahea moorei H. Bailey ex H.E. Moore

H. Chamaedorea microspadix Burret.

H. Chamaedorea sartorii Liebm.

H. Chamaedorea schiedeana Mart.

\section{DICOTYLEDONAE}

\section{ACANTHACEAE}

H. Dicliptera assurgens (L.) Juss.

H. Elytraria bromoides Oerst.

H. Justicia brandegeana Wassh. et L.B.Sm.

H. Odontonema callistachyum (Schltdl. et Cham.) Kuntze

H. Ruellia donnell-smithii Leonard

H. Ruellia sp.
Am

$\mathrm{Tr}$

Am

Am

3

TrSub

Am

Am

Am

Am 3

Am 3

Am 3

TrSub

Am 3

Am 3

Am 3

Am 3

Cos

Am

Cos

Am

Am

3

$\mathrm{Am}$

Am

Am

TrSub

Am

Am

Am

Am

2

3

TrSub

TrSub

TrSub

Am

$\operatorname{Tr}$ 


\section{AMARANTHACEAE}

$\mathrm{H}$. Achyranthes aspera L.

TrSub

B. Chamissoa altissima (Jacq.) Kunth

H. Iresine sp.

Ar. Pleuropetalum sprucei (Hook.f.) Standl.

TrSub

TrSub

$\mathrm{Am}$

\section{ANACARDIACEAE}

Ar. Rhus aromatica var. trilobata (Nutt.) A. Gray ex S. Wats.

Ar. Rhus schiedeana Schltdl.

TmSub

B. Toxicodendron radicans (L.) Kuntze

TrSub

\section{ANNONACEAE}

Ar. Annona globiflora Schltdl.

Ar. Tridimeris hahniana Baill.

$\operatorname{Tr}$

Am

1

$\begin{array}{ll}\text { Am } & 2 \\ A m & 3\end{array}$

$\operatorname{Tr}$

Cos

TrSub

A. Dendropanax arboreus (L.) Decne. et Planch.

\section{ASCLEPIADACEAE}

H. Asclepias curassavica L.

B. Blepharodon mucronatum (Schltdl.) Decne.

B. Gonolobus niger (Cav.) R.Br.

B. Marsdenia pringlei S.Watson

B. Matelea picturata (Hemsl.) Woodson

B. Matelea velutina (Schltdl.) Woodson

B. Matelea sp.

H. Metastelma angustifolium Turcz.

B. Oxypetalum cordifolium (Vent.) Schltr.

\section{BALANOPHORACEAE}

P. Helosis cayennensis (SW.) Spreng. var. mexicana (Liebm.) Hansen

BALSAMINACEAE

H. Impatiens walleriana Hook.f.

BEGONIACEAE

H. Begonia franconis Liebm.

H. Begonia glabra Aubl.

H. Begonia glandulosa A.DC. ex Hook.

H. Begonia gracilis Kunth

H. Begonia sp.

H. Begonia xilitlensis Burt-Utley

BERBERIDACEAE

Ar. Berberis sp.
$\mathrm{Tr}$

Am
Am
Am
TrSub
Am

Tr
Am

Am

TrSub

Cos 


\section{BETULACEAE}

A. Carpinus caroliniana Walter

$\mathrm{HN}$

A. Ostrya virginiana (Mill.) K.Koch

$\mathrm{HN}$

\section{BORAGINACEAE}

Ar. Cordia podocephala Torr.

Ar. Ehretia anacua (Terán et Berland.) I.M.Johnst.

H. Mimophytum omphalodoides Greenm.

Ar. Tournefortia hirsutissima L.

$\operatorname{Tr}$

TrSub

Am

TrSub

\section{CACTACEAE}

E. Aporocactus martianus (Zucc.) Britton et Rose

Am

E. Aporocactus sp.

E. Rhipsalis baccifera (S.Muell.) Stearn

$\mathrm{Tr}$

\section{CAMPANULACEAE}

H. Lobelia berlandieri A.DC. var. berlandieri

H. Lobelia divaricata Hook. et Arn.

H. Lobelia laxiflora Kunth var. laxiflora

H. Lobelia orientalis Rzed. et Calderón

H. Lobelia tarsophora Green

H. Lobelia xalapensis Kunth

H. Triodanis perfoliata (L.) Nieuwl.

$\mathrm{HN}$

\section{CAPRIFOLIACEAE}

B. Lonicera pilosa (Kunth) Spreng.

A. Sambucus mexicana C.Presl ex DC.

A. Viburnum sp.

$\mathrm{HN}$

TmSub

$\mathrm{HN}$

\section{CARYOPHYLLACEAE}

H. Arenaria lanuginosa (Michx.) Rhrb.

$\mathrm{HN}$

H. Cerastium sp.

Cos

H. Drymaria gracilis Cham. et Schltdl.

TrSub

H. Stellaria sp.

Cos

\section{CELASTRACEAE}

Ar. Elaeodendron trichotomum (Turcz.) Lundell

$\operatorname{Tr}$

$\mathrm{HN}$

A. Euonymus sp.

A. Perrottetia ovata Hemsl.

A. Wimmeria concolor Schltdl. et Cham.

TrSub

Am

\section{CLETHRACEAE}

A. Clethra kenoyeri Lundell

A. Clethra pringlei S.Watson

\section{COMPOSITAE}

H. Acmella repens (Walter) Rich.

H. Ageratina ligustrina (DC.) R.M.King et H.Rob.

H. Ageratina pazcuarensis (Kunth) R.M.King et .Rob.

TrSub

H. Ageratina petiolaris (Moc. et Sessé ex DC.) R.M.King et H.Rob.

H. Aldama dentata La Llave et Lex.

Ar. Alloispermum integrifolium (DC.) H. Rob.

H. Aster sp.

Ar. Archibaccharis schiedeana (Benth.) J.D. Jacks.

Ar. Baccharis heterophylla Kunth

Ar. Baccharis lancifolia Schicht.
Am

\section{$\operatorname{Tr}$}

Am

$\mathrm{Am}$

Cos

Am

Am 
Ar. Baccharis multiflora Kunth

H. Baccharis trinervis (Lam.) Pers.

H. Bidens alba (L.) DC. var. radiata (Sch.Bip.) Ballard Cos

H. Bidens reptans (L.) G.Don.

Ar. Brickellia glandulosa (La Llave) McVaugh Am

Ar. Calea orizabensis Klatt

$\mathrm{Am}$

Ar. Calea ternifolia Kunth

Ar. Calea urticifolia (Mill.) DC.

H. Carminatia tenuiflora DC.

H. Cirsium lappoides (Less.) Sch. Bip

Ar. Critonia hebebotrya DC.

Am

$\mathrm{HN}$

Ar. Critonia morifolia (Mill.) R.M.King et H.Rob.

Am

H. Dahlia coccinea Cav.

Am

H. Erigeron karvinskianus DC.

Cos

Ar. Eupatorium phoenicolepis B.L.Rob.

$\mathrm{HN}$

Ar. Eupatorium rivale Greenm.

Ar. Eupatorium sp.

H. Fleischmannia pycnocephala (Less.)R.M.King et H.Rob.

$\mathrm{Am}$

H. Gnaphalium sp.

Cos

H. Hieracium abscissum Less.

H. Jaegeria hirta (Lag.) Less.

TrSub

H. Lactuca graminifolia Michx. var. mexicana McVaugh

Am

Ar. Lagascea helianthifolia Kunth

Cos

H. Melampodium montanum Benth.

Am

H. Melanthera nivea (L.) Small

B. Mikania cordifolia (L.f.) Willd.

Am

$\operatorname{Tr}$

H. Milleria quinqueflora L.

$\mathrm{Tr}$

Ar. Oyedaea ovalifolia A.Gray

Ar. Piptothrix areolaris (DC.) R.M.King et H.Rob.

H. Piqueria trinervia Cav.

Ar. Pluchea carolinensis (Jacq.) G.Don

H. Pluchea symphytifolia (Mill.) Gillis

H. Polymnia maculata Cav.

H. Pseudelephantopus spicatus (Juss. ex Aubl.) C.F.Baker

B. Pseudogynoxys chenopodioides (Kunth) Cabrera

Ar. Roldana angulifolia (DC.) H.Rob. et Brettell

Ar. Roldana aschenborniana (Schauer) H.Rob. et Brettell

Am

Am

Am

Am

TrSub

Ar. Roldana lanicaulis (Greenm.) H.Rob. et Brettell

H. Sanvitalia procumbens Lam.

Am

Am

Am

Am

H. Schistocarpha bicolor Less.

H. Sclerocarpus uniserialis (Hook.) Benth. et Hook.f. ex Hemsl.

Am

Am

$\mathrm{Am}$ var. frutescens (Brandegee) Feddema.

H. Senecio argutus Kunth

H. Senecio picridis Schauer

Cos

H. Senecio tampicanus DC.

H. Stevia caracasana DC.

Ar. Stevia pilosa Lag.

H. Tagetes erecta L.

H. Tagetes filifolia Lag.

H. Tagetes lucida Cav.

H. Taraxacum officinale Weber

Ar. Tithonia longiradiata (Bertol.) S.F.Blake

H. Tridax procumbens $\mathrm{L}$.

Ar. Verbesina persicifolia DC.

Ar. Verbesina turbacensis Kunth

Ar. Vernonia alamanii DC.

Ar. Vernonia arborescens (L.) Sw.

H. Vernonia arctioides Less.

Am

TrSub

HN

Am

Am

Am

TrSub 
H. Vernonia argyropappa Buek

Ar. Vernonia heydeana J.M.Coult.

Ar. Vernonia liatroides DC.

Ar. Vernonia patens Kunth

Ar. Vernonia tortuosa (L.) S.F.Blake

Ar. Viguiera cordata (Hook. et Arn.) D'Arcy

Ar. Zexmenia sp.

Am

Am

2

CONVOLVULACEAE

H. Dichondra sericea Sw.

TrSub

B. Ipomoea indica (Burm.f.) Merr.

TrSub

B. Ipomoea sp.

B. Jacquemontia pentantha (Jacq.) G.Don f.

H. Rivea corymbosa (L.) Hallier $f$.

TrSub

TrSub

\section{CORNACEAE}

A. Cornus disciflora Moc. et Sessé ex DC.

$\mathrm{HN}$

A. Cornus excelsa Kunth

\section{CRASSULACEAE}

$\mathrm{H}$. Echeveria rosea Lindl.

H. Echeveria secunda Booth.

H. Sedum hultenii Frödin

H. Sedum moranense Kunth

H. Sedum retusum Hemsl.

H. Villadia jurgensenii (Hemsl.) Jacobsen

\section{CUCURBITACEAE}

B. Melothria pendula L.

B. Sechium edule (Jacq.) Sw.

\section{DILLENIACEAE}

A. Saurauia scabrida Hemsl.

A. Saurauia sp.

\section{ERICACEAE}

A. Arbutus xalapensis Kunth

Ar. Gaultheria trichocalcyna DC.

$\mathrm{HN}$

A. Vaccinium leucanthum Cham. et Schltdl.

TrSub

Cos

\section{EUPHORBIACEAE}

Ar. Acalypha schlechtendaliana Müll.Arg.

TrSub

H. Acalypha sp.

A. Bernardia interrupta (Schltdl.) Müll.Arg.

$\mathrm{Am}$

H. Chamaesyce nutans (Lag.) Small

Ar. Cnidosculus multilobus (Pax) I.M.Johnst.

A. Croton draco Schltdl.

A. Drypetes lateriflora (Sw.) Krug et Urb.

H. Euphorbia cyathophora Murr.

H. Euphorbia dioscoreoides Boiss.

H. Euphorbia graminea Jacq.

H. Euphorbia lasiocarpa Klotzsch.

H. Euphorbia subpeltata S.Watson

A. Gymnanthes longipes Müll.Arg.

Am

A. Gymnanthes riparia (Schltdl.) Klotzsch.

H. Phyllanthus sp.

Cos

$\mathrm{Am}$

TrSub

$\operatorname{Tr}$

Cos

Ar. Sebastiania pavoniana (Müll.Arg.) Müll.Arg.

TrSub

TrSub

H. Tragia glanduligera Pax et Hoff.

TrSub

H. Tragia sp. 
FAGACEAE

A. Quercus acutifolia Née

HN

A. Quercus affinis Scheidw.

A. Quercus aff. eugeniifolia Liebm.

A. Quercus germana Cham. et Schltdl.

A. Quercus glabrescens Benth.

A. Quercus aff. pinnativenulosa Mull.

A. Quercus polymorpha Schltdl. et Cham.

A. Quercus rysophylla Weath.

A. Quercus sartorii Liebm.

A. Quercus xalapensis Bonpl.

A. Quercus sp.

FLACOURTIACEAE

Ar. Xylosma flexuosum (Kunth) Hemsl.

A. Zuelania guidonia (Sw.) Britton et Millsp.

$\mathrm{Tr}$

Am

\section{GARRYACEAE}

A. Garrya laurifolia Benth.

Am

GERANIACEAE

H. Geranium seemannii Peyr.

Cos

GESNERIACEAE

H. Achimenes grandiflora (Schiede) DC.

H. Smithiantha zebrina (Paxton) Kuntze

Am

$\mathrm{Am}$

3

GUTTIFERAE

H. Ascyrum hypericoides L.

TrSub

HAMAMELIDACEAE

A. Liquidambar macrophylla Oerst.

HN

HYDROPHYLLACEAE

H. Nama sericeum Willd. ex Roem. et Schult.

Am

H. Nama sp.

\section{JUGLANDACEAE}

A. Carya ovata (Mill.) K.Koch var. mexicana (Engelm.)

HN W.E.Manning

A. Carya palmeri W.E.Manning

A. Juglans mollis Engelm.

HN

LABIATAE

H. Hedeoma sp.

H. Hyptis mociniana Benth.

Am

H. Hyptis mutabilis (A. Rich.) Briq.

H. Lepechinia caulescens (Ortega) Epling

H. Lepechinia schiedeana (Schltdl.) Vatke

H. Ocimum micranthum Willd.

H. Salvia coccinea L.

H. Salvia elegans Vahl

H. Salvia polystachya Ortega

H. Salvia puberula Fern. in Proc.

H. Salvia sp.

H. Scutellaria caerulea Moc. et Sessé ex Benth.

H. Scutellaria seleriana Loes.

H. Scutellaria sp.

H. Stachys albotomentosa Ramamoorthy
TrSub

Am

TrSub

TrSub

Cos

TmSub 
H. Stachys boraginoides Cham. et Schltdl.

H. Teucrium cubense Jacq.

$\operatorname{Cos}$

LAURACEAE

A. Beilschmiedia mexicana (Mez) Kosterm.

$\operatorname{Tr}$

A. Cinnamomum bractefoliaceum Lorea-Hern.

$\operatorname{Tr}$

A. Cinnamomum effusum (Meissn.) Kosterm.

A. Cinnamomum pachypodum (Nees) Kosterm.

A. Cinnamomum salicifolium (Nees) Kosterm.

A. Licaria campechiana (Standl.) Kosterm.

Ar. Litsea glaucescens Kunth

A. Nectandra salicifolia (Kunth) Nees

$\mathrm{Am}$

A. Nectandra sanguinea Rottb.

A. Ocotea klotzschiana (Nees) Hemsl.

TrSub

A. Ocotea psychotrioides Kunth

A. Persea americana Mill.

$\operatorname{Tr}$

A. Persea liebmannii Mez

\section{LEGUMINOSAE}

Ar. Amicia zygomeris DC.

A. Bauhinia chapulhuacania Wunderlin

Am

A. Bauhinia coulteri Macbr.

A. Bauhinia divaricata L.

Ar. Calliandra sp.

$\operatorname{Tr}$

$\operatorname{Tr}$

B. Canavalia villosa Benth.

$\mathrm{Tr}$

B. Canavalia sp.

H. Centrosema pubescens Benth.

$\mathrm{Am}$

H. Centrosema virginianum (L.) Benth.

A. Cercis canadensis L.

$\mathrm{HN}$

Ar. Chamaecrista nictitans (L.) Moench.

TrSub

Ar. Chamaecrista rufa (M.Martens et Galeotti) Britton et Rose

H. Cologania sp.

$\mathrm{Am}$

Am

H. Coursetia caribaea (Jacq.) Lavin

H. Crotalaria mollicula Kunth

TrSub

Ar. Crotalaria pumila Ortega

H. Crotalaria rotundifolia (Walter) Walter ex J.F.Gwel.

H. Crotalaria sagittalis L.

H. Crotalaria sp.

Ar. Dalbergia congestiflora Pittier

TrSub

A. Dalbergia glabra (Mill.) Standl.

A. Dalbergia palo-escrito Rzed. et Guridi-Gómez

Ar. Dalea lutea (Cav.) Willd.

H. Dalea scandens (Mill.) R.T.Clausen var. paucifolia (Coult.) Barneby

H. Desmodium procumbens (Mill.) A.S.Hitchc.

H. Desmodium sp.

A. Erythrina coralloides DC.

A. Erythrina sp.

H. Galactia argentea Brandegee

B. Galactia brachystachys Benth.

Ar. Indigofera cuernavacana Rose

H. Indigofera jamaicensis Spreng.

Ar. Indigofera latibracteata Harms

A. Inga lactibracteata Harms

Am

A. Inga huastecana M. Sousa

B. Lathyrus sp.

A. Leucaena pulverulenta (Schltdl.) Benth.

Am

A. Lonchocarpus caudatus Pittier

$\mathrm{Tr}$

TrSub

TrSub

TrSub

A. Lonchocarpus rugosus Benth. 
A. Lonchocarpus aff. rugosus Benth.

Ar. Mimosa albida Humb. et Bonpl. ex Willd.

TrSub

B. Mucuna cochinchinensis (Lour.) A.Chev.

$\mathrm{H}$. Nissolia fruticosa Jacq.

$\operatorname{Tr}$

Ar. Oxyrhynchus volubilis Brandegee

Am

TrSub

B. Pachyrhizus erosus (L.) Urb.

$\mathrm{Tr}$

B. Phaseolus coccineus L.

B. Phaseolus micranthus Hook. et Arn.

A. Pithecellobium insigne M. Micheli

$\mathrm{Am}$

B. Rynchosia minima (L.) DC.

Ar. Senna hirsuta (L.) H.S.Irwin et Barneby var. hirta H.S.Irwin

Am et Barneby

Ar. Senna pendula (Humb. et Bonpl. ex Willd.) H.S.Irwin et Barneby var. ovalifolia H.S.Irwin et Barneby

A. Senna racemosa (Mill.) H.S.Irwin et Barneby var. moctezumae H.S.Irwin et Barneby

B. Teramnus uncinatus (L.) Sw.

$\operatorname{Tr}$

H. Trifolium amabile Kunth

H. Vicia humilis Kunth

Ar. Zapoteca portoricensis (Jacq.) H.M. Hern.

TmSub

$\mathrm{HN}$

Am

LENTIBULARIACEAE

H. Pinguicula calderoniae Zamudio

$\mathrm{HN}$

H. Pinguicula moranensis Kunth

LINACEAE

H. Linum schiedeanum Schltdl. et Cham.

TmSub

LOASACEAE

H. Gronovia scandens L.

Am

LOGANIACEAE

A. Buddleia americana L.

TrSub

A. Buddleia cordata Kunth

A. Buddleia sessiliflora Kunth

B. Gelsemium sempervirens (L.) Pers.

H. Spigelia longiflora M.Martens et Galeotti

$\mathrm{HN}$

$\mathrm{Am}$

LORANTHACEAE

P. Phoradendron galeottii Trel.

Am

3

P. Phoradendron lanceolato-ellipticum Eichler

P. Phoradendron nervosum Oliv.

P. Phoradendron robinsonii (Urb.) Trel.

P. Phoradendron tamaulipense Trel.

P. Struthanthus quercicola (Schltdl. et Cham.) Blume

Am

3

LYTHRACEAE

H. Cuphea calaminthifolia Schldlt.

Am

H. Cuphea carthagenensis (Jacq.) Macbr.

H. Cuphea cyanea DC.

H. Cuphea lanceolata Aiton

H. Cuphea salicifolia Schltdl. et Cham.

Ar. Heimia salicifolia Link

H. Lythrum gracile Benth.

Am

Cos

MAGNOLIACEAE

A. Magnolia dealbata Zucc.

HN

A. Magnolia schiedeana Schltdl. 


\section{MALPIGHIACEAE}

B. Banisteria argentea (Kunth) Spreng.

H. Callaeum septentrionale (A.Juss.) D.M.Johnson

$\operatorname{Tr}$

Ar. Malpighia glabra L.

Ar. Tetrapteris schiedeana Schltdl. et Cham.

Am

Am

3

\section{MALVACEAE}

H. Anoda cristata (L.) Schltdl.

Am

H. Hochreutinera amplexifolia (DC.) Fryxell

Am

Am

Ar. Kearnemalvastrum lacteum (Aiton) Bates

H. Malvastrum coromandelianum (L.) Garcke

TrSub

Ar. Malvaviscus arboreus Cav.

Am

H. Pavonia schiedeana Steud.

TrSub

H. Pavonia uniflora (Sessé et Moc.) Fryxell

Ar. Phymosia umbellata Cav.

A. Robinsonella discolor Rose et E.G.Baker ex Rose

H. Sida acuta Burm.

Am 2

Am

2

H. Sida glabra Mill.

H. Sida rhombifolia L.

\section{MELASTOMATACEAE}

H. Arthrostemma ciliatum Pav. ex D.Don

Ar. Conostegia xalapensis (Bonpl.) D.Don

Ar. Leandra cornoides (Schltdl. et Cham.) Cogn.

Ar. Miconia anisotricha (Schltr.) Triana

TrSub

Ar. Miconia moorei Wurdack

Ar. Miconia sylvatica (Schltdl.) Naud.

H. Tibouchina galeottiana (Triana) Cong.

H. Tibouchina mexicana (Bonpl.) Cogn.

H. Tibouchina naudiniana (Decne.) Cogn.

Am $\quad 3$

Am 2

Am 3

$\operatorname{Tr}$

Am

\section{MELIACEAE}

A. Trichilia havanensis Jacq.

Ar. Trichilia hirta L.

Ar. Trichilia sp.

\section{MENISPERMACEAE}

B. Cissampelos pareira L.

$\mathrm{H}$. Menispermum canadense $\mathrm{L}$

$\operatorname{Tr}$

$\mathrm{HN}$

TrSub

TrSub

$\operatorname{Tr}$

$\operatorname{Cos}$

Ar. Myrica cerifera L.

MYRSINACEAE

Ar. Ardisia escallonioides Schltdl. et Cham.

TrSub

Am

Am

1

Ar. Parathesis brevipes Lundell

Ar. Parathesis leptopa Lundell

Ar. Parathesis serrulata (Sw.) Mez

Ar. Parathesis subcoriacea Lundell

A. Rapanea myricoides (Schltdl.) Lundell

TrSub

MYRTACEAE

A.Eugenia capuli (Schltdl. et Cham.) O.Bergman

MORACEAE

A. Ficus pertusa $\mathrm{L}$.

A. Morus celtidifolia Kunth

Ar. Trophis racemosa (L.) Urb.

3
2 3 3 3 
A. Eugenia crenularis Lundell

A. Eugenia xalapensis (Kunth) DC.

A.Eugenia xilitensis (Kunth) DC.

A. Myrcianthes fragrans (Sw.) McVaugh

$\mathrm{Am}$

NYCTAGINACEAE

H. Mirabilis jalapa L.

Am

OLACACEAE

Ar. Schoepfia schreberi J.F.Gmel.

$\operatorname{Tr}$

OLEACEAE

A. Forestiera reticulata Torr.

A. Fraxinus uhdei (Wenz.) Lingelsh.

A. Osmanthus americana (L.) Benth. et Hook.

Am

$\mathrm{HN}$

ONAGRACEAE

H. Fuchsia encliandra Steud.

$\mathrm{HN}$

H. Fuchsia sp.

H. Lopezia miniata (DC.) Lag.

TrSub

H. Lopezia racemosa Cav.

H. Oenothera rosea L'Hér.

H. Oenothera sp.

Am

OROBANCHACEAE

P. Conopholis alpina Liebm.

Am

OXALIDACEAE

H. Oxalis acuminata Schltdl. et Cham.

$\operatorname{Cos}$

H. Oxalis corniculata L.

Ar. Oxalis rhombifolia Jacq.

PAPAVERACEAE

$\mathrm{H}$. Argemone grandiflora Sweet

Am

$\mathrm{H}$. Argemone mexicana L.

Ar. Bocconia frutescens $\mathrm{L}$.

H. Corydalis pseudomicrantha Fedde

Am

$\mathrm{HN}$

PASSIFLORACEAE

B. Passiflora capsularis L.

TrSub

B. Passiflora serratifolia L.

B. Passiflora sicyoides Schltdl. et Cham.

PHYTOLACCACEAE

H. Phytolacca purpurascens A.Braun et Bouché

TrSub

Ar. Phytolacca rivinoides Kunth et Bouché

H. Rivina humilis L.

B. Trichostigma octandrum (L.) H.Walter

Am

Am

\section{PIPERACEAE}

H. Peperomia blanda (Jacq.) Kunth

TrSub

E. Peperomia collocata Trel.

H. Peperomia galioides Kunth

E. Peperomia microphylla Kunth

E. Peperomia quadrifolia (L.) Kunth

Ar. Pothomorphe umbellata (L.) Miq.

Ar. Piper auritum Kunth

$\operatorname{Tr}$

Ar. Piper fraguanum Trel.

$\operatorname{Tr}$

PLANTAGINACEAE

H. Plantago australis Lam.

Cos 
POLEMONIACEAE

B. Cobaea stipularis Benth.

H. Gilia incisa Benth.

H. Loeselia glandulosa (Cav.) G.Don

$\mathrm{HN}$

$\mathrm{Am}$

\section{POLYGALACEAE}

Ar. Monnina xalapensis Kunth

H. Polygala aparinoides Hook. et Arn.

$\mathrm{Am}$

H. Polygala paniculata L.

Cos

\section{POLYGONACEAE}

H. Antigonon leptopus Hook. et Arn.

Am

A. Coccoloba barbadensis Jaca.

H. Polygonum lapathifolium L.

Am

Cos

PRIMULACEAE

$\mathrm{H}$. Anagallis arvensis L.

Cos

PROTEACEAE

A. Roupala montana Aubl.

PYROLACEAE

H. Chimaphila maculata (L.) Pursh

Cos

\section{RANUNCULACEAE}

B. Clematis pitcheri Torr. et A.Gray

TmSub

H. Thalictrum strigillosum Hemsl.

TrSub

RHAMNACEAE

Ar. Ceanothus coeruleus Lag.

Ar. Colubrina greggii S.Watson

Am

Ar. Gouania polygama (Jacq.) Urb.

Am

A. Rhamnus capreaefolia Schltdl.

TrSub

A. Rhamnus serrata Humb. et Bonpl. ex Schult.

TrSub

\section{ROSACEAE}

$\mathrm{H}$. Alchemilla pectinata Kunth

$\mathrm{HN}$

A. Cercocarpus macrophyllus Schneid.

H. Duchesnea indica (Andrews) Facke

Am

TrSub

A. Photinia mexicana (Baill.) Hemsl.

TrSub

Ar. Prunus samydoides Schltdl.

$\mathrm{HN}$

A. Prunus serotina Ehrenb. ssp. serotina

\section{RUBIACEAE}

H. Borreria laevis (Lam.) Griseb.

H. Bouvardia ternifolia (Cav.) Schltdl.

TrSub

H. Bouvardia xylosteoides Hook. et Arn.

Ar. Chiococca alba (L.) Hitchc.

Ar. Chiococca pachyphylla Wernham

Ar. Chione mexicana Standl.

H. Coccocypselum guianense (Aubl.) K.Schum.

$\mathrm{Am}$

H. Crusea longiflora (Willd. ex Roem et Schult.) W.R.Anderson

\section{$\operatorname{Tr}$}

Am

H. Deppea purpusii Standl.

$\mathrm{Am}$

H. Galium orizabense Hemsl.

Am

H. Galium uncinulatum DC.

Ar. Hamelia patens Jacq.

Cos

H. Hedyotis watsonii W.H.Lewis

Am

TrSub

Ar. Hoffmannia sp.

H. Mitchella repens L.

H. Nertera granadensis (L.) Druce

Am

$\mathrm{HN}$

TrSub 
Ar. Psychotria erythrocarpa Schltdl.

Ar. Psychotria graciliflora Benth. ex Oerst.

Ar. Psychotria microdon (DC.) Urb.

H. Psychotria pubescens Sw.

Ar. Psychotria tenuifolia Sw.

Ar. Randia sp.

Ar. Rondeletia heteranthera Brandegee

$\operatorname{Tr}$

Am

\section{RUTACEAE}

Ar. Choisya ternata Kunth

Ar. Decatropis bicolor (Zucc.) Radlk.

Ar. Zanthoxylum elegantissimum (Engl.) P.Wilson

A. Zanthoxylum fagara (L.) Sarg.

A. Zanthoxylum hidalgense Lundell

\section{SAPINDACEAE}

A. Cupania dentata DC.

B. Paullinia tomentosa Jacq.

A. Sapindus saponaria L.

B. Serjania sp.

Am

$\mathrm{Am}$

Cos

Am

Am

TrSub

$\mathrm{Am}$

3

SAPOTACEAE

Ar. Bumelia verruculosa Cronquist

Ar. Chrysophyllum mexicanum Brandegee ex Standl.

Am

$\operatorname{Tr}$

\section{SAXIFRAGACEAE}

Ar. Hydrangea aff. nebulicola Nevl. et Gómez Pompa

HN

B. Philadelphus affinis Schltdl.

B. Philadelphus coulteri Watson

$\mathrm{HN}$

SCROPHULARIACEAE

H. Bacopa procumbens (Mill.) Greenm.

TrSub

H. Capraria saxifragaefolia Schltdl. et Cham.

Ar. Lamourouxia multifida Kunth

H. Leucocarpus perfoliatus (Kunth) Benth.

Am

Am

H. Maurandya erubescens (D.Don) Gray

Am

3

H. Melasma physalodes (D.Don) Melch.

H. Penstemon barbatus (Cav.) Roth

$\mathrm{HN}$

$\operatorname{Tr}$

Am

H. Russelia coccinea (L.) Wettst.

Am

H. Russelia maculosa Lundell

H. Russelia syringaefolia Schltdl. et Cham.

H. Russelia sp.

H. Veronica persica Poir.

HN

\section{SIMAROUBACEAE}

Ar. Picramnia andicola Tul.

$\mathrm{Am}$

Ar. Picramnia antidesma Sw.

Ar. Picramnia xalapensis Planch.

\section{SOLANACEAE}

H. Capsicum ciliatum (Kunth) Kuntze

Am

3

Ar. Cestrum oblongifolium Schltdl.

$\mathrm{Am}$

Am

Ar. Datura candida (Pers.) Saff.

H. Jaltomata procumbens (Cav.) J.L.Gentry

Am

H. Physalis gracilis Miers

Cos

H. Physalis melanocystis (B.L.Rob.) Bitter

H. Physalis queretaroensis M. Martínez et L. Hernández

H. Physalis pubescens L.

H. Physalis sordida Fernald

B. Solandra nitida Zucc. 
Ar. Solanum acerifolium Dunal

Cos

H. Solanum americanum Mill.

Ar. Solanum aphyodendron S.Knapp

B. Solanum appendiculatum Dunal

Ar. Solanum diphyllum L.

Ar. Solanum erianthum D.Don

Ar. Solanum hispidum Pers.

B. Solanum iopetalum (Bitter) Hawkes

Ar. Solanum lanceifolium Jacq.

H. Solanum myriacanthum Dunal

Ar. Solanum nigrescens M.Martens et Galeotti

Ar. Solanum nudum Dunal

H. Solanum seudocapsicum Vell.

H. Solanum pubescens Ruiz et Pav.

H. Solanum refractum Hook. et Arn.

A. Solanum salviifolium Lam.

A. Solanum schlechtendalianum Walp.

H. Solanum seaforthianum Andr.

B. Solanum skutchii Correll

Ar. Solanum umbellatum Mill.

Ar. Witheringia mexicana (B.L.Rob.) Hunz.

Am

Ar. Witheringia solanacea L'Hér.

Ar. Witheringia stellata (Greenm.) Hunz.

\section{STAPHYLEACEAE}

A. Turpinia occidentalis (Sw.) G.Don

TrSub

STERCULIACEAE

A. Reevesia clarkii (Monach. et Moldenke) Solheim TrSub

STYRACACEAE

A. Styrax glabrescens Benth.

TrSub

THEACEAE

A. Cleyera theaeoides (Sw.) Choisy

A. Ternstroemia huasteca B.M.Barthol.

A. Ternstroemia sylvatica Schltdl. et Cham.

THYMELAEACEAE

A. Daphnopsis mollis (Cham. et Schltdl.) Standl.

\section{TILIACEAE}

H. Corchorus siliquosus L.

A. Heliocarpus americanus L.

A. Heliocarpus appendiculatus Turcz.

A. Heliocarpus mexicanus (Turcz.) Sprague

A. Tilia mexicana Schltdl.

Ar. Triumfetta grandiflora Vahl

$\mathrm{HN}$

Ar. Triumfetta semitriloba Jacq.

$\operatorname{Tr}$

Ar. Triumfetta sp.

\section{ULMACEAE}

A. Aphananthe monoica (Hemsl.) Leroy

Ar. Celtis laevigata Willd.

TrSub

$\mathrm{Tr}$

Ar. Celtis iguanaea (Jacq.) Sarg.

A. Trema micrantha (L.) Blume

TrSub

A.Ulmus mexicana (Liebm.) Planch.

$\mathrm{HN}$

UMBELLIFERAE

H. Arracacia tolucensis (Kunth) Hemsl. 
H. Coulterophytum brevipes J.M.Coult. et Rose

$\mathrm{Am}$

H. Coulterophytum laxum B.L.Rob.

H. Eryngium nasturtiifolium Juss. ex F.Delaroche

TrSub

H. Hydrocotyle mexicana Cham et. Schltdl.

H. Sanicula liberta Cham. et Schltdl.

$\mathrm{Tr}$

H. Spananthe paniculata Jacq.

Cos

$\mathrm{Am}$

\section{URTICACEAE}

A. Boehmeria ulmifolia Wedd

TrSub

Ar. Myriocarpa longipes Liebm.

Am

H. Parietaria pensylvanica (Muehl.) Willd.

H. Phenax hirtus (Sw.) Weed

Cos

H. Pilea acuminata Liebm.

$\mathrm{Am}$

H. Pilea microphylla (L.) Liebm.

TrSub

H. Pilea pubescens Liebm.

Ar. Urera alceifolia Gaudich.

TrSub

Ar. Urera caracasana (Jacq.) Griseb

H. Urtica mexicana Liebm.

Cos

VALERIANACEAE

H. Valeriana clematitis Kunth

Cos

B. Valeriana scandens $\mathrm{L}$.

\section{VERBENACEAE}

Ar. Callicarpa acuminata Kunth

TrSub

Ar. Callicarpa pringlei Briq.

A. Citharexylum glabrum (S.Watson) Greenm.

Am

Ab. Citharexylum hidalgense Moldenke

Ar. Clerodendrum bungei Steud.

TrSub

Ar. Clerodendrum fragrans Vent.

H. Lantana camara L.

$\operatorname{Tr}$

Ar. Lantana hirta Graham

Ar. Lantana involucrata L.

Ar. Lippia myriocephala Schltdl. et Cham.

H. Lippia strigulosa Martens et Galeotti

Ar. Lippia sp.

H. Petrea volubilis L.

H. Priva aspera Kunth

H. Priva lappulacea (L.) Pers.

H. Priva mexicana (L.) Pers.

H. Stachytarpheta purpurea Greenm. TrSub

H. Verbena amoena Paxton

TrSub

H. Verbena delticola Small

H. Verbena elegans Kunth

H. Verbena litoralis Kunth

H. Verbena sp.

\section{VIOLACEAE}

H. Hybanthus attenuatus (Humb. et Bonpl.) G.K.Schulze

TrSub

Ar. Hybanthus mexicanus Ging.

Am

\section{VITACEAE}

B. Cissus rhombifolia Vahl

TrSub

B. Cissus sicyoides L.

B. Parthenocissus quinquefolia (L.) Planch.

B. Vitis bourgaeana Planch.

TmSub

B. Vitis popenoei J.H.Fennell

$\mathrm{HN}$

B. Vitis tiliifolia Humb. et Bonpl. ex Roem et Schult. 